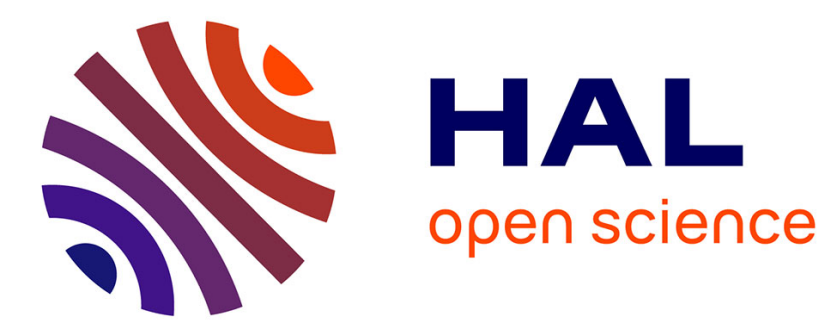

\title{
Building up time-consistency for risk measures and dynamic optimization
}

Michel de Lara, Vincent Leclère

\section{To cite this version:}

Michel de Lara, Vincent Leclère. Building up time-consistency for risk measures and dynamic optimization. European Journal of Operational Research, 2015, 10.1016/j.ejor.2015.03.046 . hal01208297

\section{HAL Id: hal-01208297 \\ https://hal-enpc.archives-ouvertes.fr/hal-01208297}

Submitted on 2 Oct 2015

HAL is a multi-disciplinary open access archive for the deposit and dissemination of scientific research documents, whether they are published or not. The documents may come from teaching and research institutions in France or abroad, or from public or private research centers.
L'archive ouverte pluridisciplinaire HAL, est destinée au dépôt et à la diffusion de documents scientifiques de niveau recherche, publiés ou non, émanant des établissements d'enseignement et de recherche français ou étrangers, des laboratoires publics ou privés. 


\title{
Building up Time-Consistency for Risk Measures and Dynamic Optimization
}

\author{
Michel De Lara ${ }^{\mathrm{a}}$, Vincent Leclère ${ }^{\mathrm{a}, *}$ \\ ${ }^{a}$ Université Paris-Est, CERMICS (ENPC), 6-8 Avenue Blaise Pascal, Cité Descartes , \\ F-77455 Marne-la-Vallée
}

\begin{abstract}
In stochastic optimal control, one deals with sequential decision-making under uncertainty; with dynamic risk measures, one assesses stochastic processes (costs) as time goes on and information accumulates. Under the same vocable of time-consistency (or dynamic-consistency), both theories coin two different notions: the latter is consistency between successive evaluations of a stochastic processes by a dynamic risk measure (a form of monotonicity); the former is consistency between solutions to intertemporal stochastic optimization problems. Interestingly, both notions meet in their use of dynamic programming, or nested, equations.

We provide a theoretical framework that offers i) basic ingredients to jointly define dynamic risk measures and corresponding intertemporal stochastic optimization problems ii) common sets of assumptions that lead to time-consistency for both. We highlight the role of time and risk preferences - materialized in one-step aggregators - in time-consistency. Depending on how one moves from one-step time and risk preferences to intertemporal time and risk preferences, and depending on their compatibility (commutation), one will or will not observe time-consistency. We also shed light on the relevance of information structure by giving an explicit role to a state control dynamical system, with a state that parameterizes risk measures and is the input to optimal policies.
\end{abstract}

Keywords: dynamic programming, time-consistency, dynamic risk measures

\section{Introduction}

You come accross time-consistency in two different mathematical fields. You are time-consistent if, as time goes on and information accumulates, you do not question the original assessment of stochastic processes (dynamic risk measures) 5 or planning of policies (stochastic optimal control).

\footnotetext{
${ }^{*}$ Corresponding author

Email address: vincent.leclere@cermics.enpc.fr (Vincent Leclère)
} 
We propose a general mechanism to build up time-consistent dynamic risk measures, that serve as criteria for optimal control problems under uncertainty, which henceforth inherit time-consistency. We show how in a few words.

Consider two sets $\mathbb{T}_{1}$ and $\mathbb{T}_{2}$, representing sets of time periods $\left(\mathbb{T}_{1}=\{1,2,3\}\right.$, $\mathbb{T}_{2}=\{4,5\}$ for instance). Consider two sets $\mathbb{W}_{1}$ and $\mathbb{W}_{2}$, representing possible values of uncertainties. For any set $\mathbb{S}$, denote by $\mathcal{L}(\mathbb{S})$ the set of functions $\mathbb{S} \rightarrow \mathbb{R} \cup\{+\infty\}$, and by $\mathbb{G}_{\mathbb{S}}: \mathcal{L}(\mathbb{S}) \rightarrow \mathbb{R} \cup\{+\infty\}$ a mapping. You can assess any function $A: \mathbb{T}_{1} \times \mathbb{T}_{2} \times \mathbb{W}_{1} \times \mathbb{W}_{2} \rightarrow \mathbb{R} \cup\{+\infty\}$,

- either by block-aggregation: start by aggregating by time, yielding $\mathbb{G}_{\mathbb{T}_{2}} \mathbb{G}_{\mathbb{T}_{1}}$ : $\mathbb{W}_{1} \times \mathbb{W}_{2} \rightarrow \mathbb{R} \cup\{+\infty\}$, then by uncertainty, yielding $\mathbb{G}_{\mathbb{W}_{2}} \mathbb{G}_{\mathbb{W}_{1}} \mathbb{G}_{\mathbb{T}_{2}} \mathbb{G}_{\mathbb{T}_{1}} A \in$ $\mathbb{R} \cup\{+\infty\}$,

- or by nested-aggregation, yielding $\mathbb{G}_{\mathbb{W}_{2}} \mathbb{G}_{\mathbb{T}_{2}} \mathbb{G}_{\mathbb{W}_{1}} \mathbb{G}_{\mathbb{T}_{1}} A \in \mathbb{R} \cup\{+\infty\}$.

We will show that nested-aggregation produces both time-consistent dynamic risk measures and optimal control problems, and that so does block-aggregation when a commutation property holds true. For example sum and integral are commuting operators and a block-aggregation is equivalent to a nested-aggregation as shown in the following equality

$$
\begin{aligned}
\iiint_{X \times Y \times Z}\left[c_{1}(x)+c_{2}(x, y)\right. & \left.+c_{3}(x, y, z)\right] d x d y d z= \\
& \int_{X}\left[c_{1}(x)+\int_{Y}\left[c_{2}(x, y)+\int_{Z} c_{3}(x, y, z) d x\right] d y\right] d z .
\end{aligned}
$$

Now, let us be more specific.

In stochastic optimal control, one deals with sequential decision-making under uncertainty; with dynamic risk measures, one assesses stochastic processes (costs) as time goes on and information accumulates. We discuss the definition of time-consistency in each setting one after the other (see also [1] for another analysis of links between both notion).

In optimal control problems, we consider a dynamical process that can be influenced by exogenous noises as well as decisions made at every time step. The decision-maker (DM) wants to optimize a criterion (for instance, minimize a net present value) over a given time horizon. As time goes on and the system evolves, the DM makes observations. Naturally, it is generally more profitable for the DM to adapt his decisions to the observations. He is hence looking for 30 policies (strategies, decision rules) rather than simple decisions: a policy is a function that maps every possible history of the observations to corresponding decisions.

The notion of "consistent course of action" (see [2]) is well-known in the field of economics, with the seminal work of [3] : an individual having planned 35 his consumption trajectory is consistent if, reevaluating his plans later on, he does not deviate from the originally chosen plan. This idea of consistency as "sticking to one's plan" may be extended to the uncertain case where plans are 
replaced by decision rules ("Do thus-and-thus if you find yourself in this portion of state space with this amount of time left", Richard Bellman cited in [4): [5] 40 addresses "consistency" and "coherent dynamic choice", 6] refers to "temporal consistency".

In this context, we loosely state the property of time-consistency in optimal control problems as follows [7. The decision maker formulates an optimization problem at time $t_{0}$ that yields a sequence (planning) of optimal decision rules

45 for $t_{0}$ and for the following increasing time steps $t_{1}, \ldots, t_{N}=T$. Then, at the next time step $t_{1}$, he formulates a new problem starting at $t_{1}$, that yields a new sequence of optimal decision rules from time steps $t_{1}$ to $T$. Suppose the process continues until time $T$ is reached. The sequence of optimization problems is said to be time-consistent if the optimal strategies obtained when solving the so original problem at time $t_{0}$ remain optimal for all subsequent problems. In other words, time consistency means that strategies obtained by solving the problem at the very first stage do not have to be questioned later on.

Now, we turn to dynamic risk measures. At time $t_{0}$, you assess, by means of a risk measure $\rho_{t_{0}, T}$, the "risk" of a stochastic process $\left\{\boldsymbol{A}_{t}\right\}_{t=t_{0}}^{t_{N}}$, that represents 55 a stream of costs indexed by the increasing time steps $t_{0}, t_{1}, \ldots, t_{N}=T$. Then, at the next time step $t_{1}$, you assess the risk of the tail $\left\{\boldsymbol{A}_{t}\right\}_{t=t_{1}}^{t_{N}}$ of the stochastic process knowing the information obtained and materialized by a $\sigma$-field $\mathfrak{F}_{t_{1}}$. For this, you use a conditional risk measure $\rho_{t_{1}, T}$ with values in $\mathfrak{F}_{t_{1}}$-measurable random variables. Suppose the process continues until time $T$ is reached. The sequence $\left\{\rho_{t, T}\right\}_{t=t_{0}}^{t_{N}}$ of conditional risk measures is called a dynamic risk measure.

Dynamic or time-consistency has been introduced in the context of risk measures (see [8, 9, 10, 11, 12] for definitions and properties of coherent and consistent dynamic risk measures). The dynamic risk measure $\left\{\rho_{t, T}\right\}_{t=t_{0}}^{t_{N}}$ is said to be ${ }_{65}$ time-consistent when the following property holds. Suppose that two streams of costs, $\left\{\underline{\boldsymbol{A}}_{t}\right\}_{t=t_{0}}^{t_{N}}$ and $\left\{\overline{\boldsymbol{A}}_{t}\right\}_{t=t_{0}}^{t_{N}}$, are such that they coincide from time $t_{i}$ up to time $t_{j}>t_{i}$ and that, from that last time $t_{j}$, the risk of the tail stream $\left\{\underline{\boldsymbol{A}}_{t}\right\}_{t=t_{j}}^{t_{N}}$ is more than that of $\left\{\overline{\boldsymbol{A}}_{t}\right\}_{t=t_{j}}^{t_{N}}$ (i.e. $\left.\rho_{t_{j}, T}\left(\left\{\underline{\boldsymbol{A}}_{t}\right\}_{t=t_{j}}^{t_{N}}\right) \geq \rho_{t_{j}, T}\left(\left\{\overline{\boldsymbol{A}}_{t}\right\}_{t=t_{j}}^{t_{N}}\right)\right)$. Then, the whole stream $\left\{\underline{\boldsymbol{A}}_{t}\right\}_{t=t_{i}}^{t_{N}}$ has higher risk than $\left\{\overline{\boldsymbol{A}}_{t}\right\}_{t=t_{i}}^{t_{N}}$ (i.e. $\rho_{t_{i}, T}\left(\left\{\underline{\boldsymbol{A}}_{t}\right\}_{t=t_{i}}^{t_{N}}\right) \geq$ $\left.\rho_{t_{i}, T}\left(\left\{\overline{\boldsymbol{A}}_{t}\right\}_{t=t_{i}}^{t_{N}}\right)\right)$.

We observe that both notions of time-consistency look quite different: the latter is consistency between successive risk assessments of a stochastic process by a dynamic risk measure (a form of monotonicity); the former is consistency between solutions to intertemporal stochastic optimization problems. We now 75 stress the role of information accumulation in both notions of time-consistency, because it highlights how the two notions can be connected. For dynamic risk measures, the flow of information is materialized by a filtration $\left\{\mathfrak{F}_{t}\right\}_{t=t_{1}}^{t_{N}}$. In stochastic optimal control, an amount of information more modest than the past of exogenous noises is often sufficient to make an optimal decision. In 
the seminal work of [13, the minimal information necessary to make optimal decisions is captured in a state variable (see [14 for a more formal definition). Moreover, the famous Bellman or Dynamic Programming Equation (DPE) provides a theoretical way to find optimal strategies (see [15] for a broad overview on Dynamic Programming (DP)).

Interestingly, time-consistency in optimal control problems and time-consistency for dynamic risk measures meet in their use of DPEs. On the one hand, in optimal control problems, it is well known that the existence of a DPE with state $x$ for a sequence of optimization problems implies time-consistency when solutions are looked after as feedback policies that are functions of the state $x$. On the other hand, proving time-consistency for a dynamic risk measure appears rather easy when the corresponding conditional risk measures can be expressed by a nested formulation. In both contexts, such nested formulations are possible only for proper information structures. In optimal control problems, a sequence of optimization problems may be consistent for some information structure while 95 inconsistent for a different one (see [7]). For dynamic risk measures, timeconsistency appears to be strongly dependent on the underlying information structure (filtration or scenario tree). Moreover, in both contexts, nested formulations and the existence of a DPE are established under various forms of decomposability of operators that display monotonicity and commutation properties.

Our objective is to provide a theoretical framework that offers i) basic ingredients to jointly define dynamic risk measures and corresponding intertemporal optimization problems under uncertainty ii) common sets of assumptions that lead to time-consistency for both. We wish to highlight the role of time and risk 105 preferences, materialized in one-step aggregators, in time-consistency. Depending on how you move from one-step time and risk preferences to intertemporal time and risk preferences, and depending on their compatibility (commutation), you will or will not observe time-consistency. We also shed light on the relevance of information structure by giving an explicit role to a dynamical system with state $x$.

The paper is organized as follows. In $\$ 2$, we define dynamic uncertainty criteria ("cousins" of dynamic risk measures) and their time-consistency. Then, we introduce the notions of time and uncertainty-aggregators, define their composition, and show two ways to craft a dynamic uncertainty criterion from one${ }_{115}$ step aggregators: in the nested-aggregation case, we prove time-consistency; in the block-aggregation case, we have to add a commutation property for this. In 43 , we introduce the basic material to formulate intertemporal optimization problems under uncertainty from dynamic uncertainty criteria, and define their time-consistency. In the nested-aggregation case, we prove time-consistency by displaying a DPE; in the block-aggregation case, we have to add a commutation property for this. We end with applications in 4 , before concluding.

\section{Notations}

We fix notations used throughout the paper: 
- $\llbracket a, b \rrbracket$ is the set of integers between $a$ and $b$ (included);

- $\left\{u_{t}\right\}_{0}^{T}$ is the sequence $\left\{u_{0}, \ldots, u_{T}\right\}$;

- $\overline{\mathbb{R}}=\mathbb{R} \cup\{+\infty\}$

- $\mathbb{W}_{[0: s]}$ is the Cartesian product $\mathbb{W}_{0} \times \cdots \times \mathbb{W}_{s}$;

- $\mathbb{G}$ is used to refer to an aggregator with respect to uncertainty;

- $\phi$ is used to refer to an aggregator with respect to time.

Furthermore, the superscript notation indicates that the domain of the mapping $\mathbb{G}^{[t: s]}$ is $\mathcal{F}\left(\mathbb{W}_{[t: s]} ; \overline{\mathbb{R}}\right)$ (not to be confused with $\mathbb{G}_{[t: s]}=\left\{\mathbb{G}_{r}\right\}_{r=t}^{s}$ ).

\section{Building up Time-Consistent Risk Measures}

In 2.1. we lay out adapted uncertainty processes and dynamic uncertainty criterion, cousins of adapted processes and dynamic risk measures, then propose a definition of time-consistency. Then, in $\$ 2.2$ we introduce the notions of time and uncertainty-aggregators, define their composition, and outline general ways of building a dynamic uncertainty criterion from one-step aggregators. Further, in $\$ 2.3$ we define nested criterion and give a time-consistency result relying on monotonicity of one-step aggregators. Finally, in 2.4 we define a commutation property between time and uncertainty aggregators. This commutation property allow to give a time-consistency result for a block-aggregated dynamic uncertainty criterion.

\subsection{Time-Consistent Dynamic Uncertainty Criterion}

145 Inspired by the definitions of risk measures and dynamic risk measures in Mathematical Finance, and motivated by intertemporal optimization, we introduce the following definitions of dynamic uncertainty criterion, and Markov dynamic uncertainty criterion. Such criteria are not restricted to assess measurable mappings (as stochastic processes would be), and we pay the price by letting $+\infty$ be a possible assessment (for instance, the "mathematical expectation" of a non-measurable function is $+\infty$ ).

Mimicking the definition of adapted processes in probability theory, we first introduce the following definition of adapted uncertainty processes.

Definition 1. We say that a sequence $A_{[0: T]}=\left\{A_{s}\right\}_{0}^{T}$ is an adapted uncertainty process if $A_{s} \in \mathcal{F}\left(\mathbb{W}_{[0: s]} ; \overline{\mathbb{R}}\right)$ (that is, $A_{s}: \mathbb{W}_{[0: s]} \rightarrow \overline{\mathbb{R}}$ ), for all $s \in \llbracket 0, T \rrbracket$. In other words, $\left[\mathcal{F}\left(\mathbb{W}_{[0: s]} ; \overline{\mathbb{R}}\right)\right]_{s=0}^{T}$ is the set of adapted uncertainty processes.

Definition 2. A dynamic uncertainty criterion is a sequence $\left\{\varrho_{t, T}\right\}_{t=0}^{T}$, such that, for all $t \in \llbracket 0, T \rrbracket$, 
- $\varrho_{t, T}$ is a mapping

$$
\varrho_{t, T}:\left[\mathcal{F}\left(\mathbb{W}_{[0: s]} ; \overline{\mathbb{R}}\right)\right]_{s=t}^{T} \rightarrow \mathcal{F}\left(\mathbb{W}_{[0: t]} ; \overline{\mathbb{R}}\right),
$$

- the restriction of $\varrho_{t, T}$ to the domain $1\left[\mathcal{F}\left(\mathbb{W}_{[t: s]} ; \overline{\mathbb{R}}\right)\right]_{s=t}^{T}$ yields constant functions, that is,

$$
\varrho_{t, T}:\left[\mathcal{F}\left(\mathbb{W}_{[t: s]} ; \overline{\mathbb{R}}\right)\right]_{s=t}^{T} \rightarrow \overline{\mathbb{R}}
$$

A Markov dynamic uncertainty criterion is a dynamic uncertainty criterion parametrized at each step $t$ by a state $x_{t}$ belonging to a state space $\mathbb{X}_{t}$.

We establish a parallel between uncertainty criteria and risk measures. For this purpose, when needed, we implicitely suppose that each uncertainty set $\mathbb{W}_{t}$ is endowed with a $\sigma$-algebra $\mathcal{W}_{t}$, so that the set $\mathbb{W}_{[0: T]}$ of scenarios is naturally equipped with the filtration

$$
\mathfrak{F}_{t}=\mathcal{W}_{0} \otimes \cdots \otimes \mathcal{W}_{t} \otimes\left\{\emptyset, \mathbb{W}_{t+1}\right\} \otimes \cdots \otimes\left\{\emptyset, \mathbb{W}_{T}\right\}, \quad \forall t \in \llbracket 0, T \rrbracket .
$$

Notice that, when the $\sigma$-algebra $\mathcal{W}_{t}$ is the complete $\sigma$-algebra made of all subsets of $\mathbb{W}_{t}, \mathcal{F}\left(\mathbb{W}_{[0: t]} ; \overline{\mathbb{R}}\right)$ is exactly the space of random variables that are $\mathfrak{F}_{t^{-}}$ measurable.

We provide a definition of time-consistency for Markov dynamic uncertainty criteria, inspired by the definition for dynamic risk measures. If two streams of random costs coincide up to time $t$, but that the tail streams can be ranked pointwise, so will their risk measure.

Definition 3. The Markov dynamic uncertainty criterion $\left\{\left\{\varrho_{t, T}^{x_{t}}\right\}_{x_{t} \in \mathbb{X}_{t}}\right\}_{t=0}^{T}$ is 170 said to be time-consistent if, for any couple of times $0 \leq \underline{t}<\bar{t} \leq T$, the following property holds true.

If two adapted uncertainty processes $\left\{\underline{A}_{s}\right\}_{0}^{T}$ and $\left\{\bar{A}_{s}\right\}_{0}^{T}$, satisfy

$$
\begin{aligned}
\underline{A}_{s} & =\bar{A}_{s}, & \forall s \in \llbracket \underline{t}, \bar{t} \rrbracket, \\
\rho_{\bar{t}, T}^{\bar{x}}\left(\left\{\underline{A}_{s}\right\}_{\bar{t}}^{T}\right) & \leq \rho_{\bar{t}, T}^{\bar{x}}\left(\left\{\bar{A}_{s}\right\}_{\bar{t}}\right), & \forall \bar{x} \in \mathbb{X}_{\bar{t}},
\end{aligned}
$$

then we have:

$$
\rho_{\underline{\underline{t}}, T}^{\underline{x}}\left(\left\{\underline{A}_{s}\right\}_{\underline{\underline{t}}}^{T}\right) \leq \rho_{\underline{\underline{t}}, T}^{\underline{x}}\left(\left\{\bar{A}_{s}\right\}_{\underline{\underline{t}}}^{T}\right), \quad \forall \underline{x} \in \mathbb{X}_{\underline{t}} .
$$

\subsection{Aggregators and their Composition}

We introduce the notions of time and uncertainty-aggregators, define their composition, and outline general ways of constructing a dynamic uncertainty criterion from one-step aggregators.

\footnotetext{
${ }^{1}$ Where $\mathcal{F}\left(\mathbb{W}_{[t: s]} ; \overline{\mathbb{R}}\right)$ is naturally identified as a subset of $\mathcal{F}\left(\mathbb{W}_{[0: s]} ; \overline{\mathbb{R}}\right)$.
} 


\subsubsection{One-Step Time-Aggregators and their Composition}

For notational clarity the argument of time-aggregators are written between curly braces $(\{\cdot\})$ whereas the argument of uncertainty aggregators are written between straight brackets [·].

Definition 4. A multiple-step time-aggregator is a function mapping $\overline{\mathbb{R}}^{k}$ into $\overline{\mathbb{R}}$, where $k \geq 2$. When $k=2$, we call one-step time-aggregator a function mapping $\overline{\mathbb{R}}^{2}$ into $\overline{\mathbb{R}}$. A one-step time-aggregator is said to be non-decreasing if it is non-decreasing in its second variable.

Let $\Phi^{1}: \overline{\mathbb{R}}^{2} \rightarrow \overline{\mathbb{R}}$ be a one-step time-aggregator and $\Phi^{k}: \overline{\mathbb{R}}^{k} \rightarrow \overline{\mathbb{R}}$ be a multiple-step time-aggregator. We define the composition $\Phi^{1} \odot \Phi^{k}: \overline{\mathbb{R}}^{k+1} \rightarrow \overline{\mathbb{R}}$ by

$$
\left(\Phi^{1} \odot \Phi^{k}\right)\left\{c_{1}, c_{2}, \ldots, c_{k+1}\right\}=\Phi^{1}\left\{c_{1}, \Phi^{k}\left\{c_{2}, \ldots, c_{k+1}\right\}\right\} .
$$
by

The composition of multiple one-step time-aggregator is defined recursively

$$
\left(\bigodot_{s=t}^{T-1} \Phi_{s}\right)\left(c_{[t: T]}\right)=\Phi_{t}\left\{c_{t},\left(\bigodot_{s=t+1}^{T-1} \Phi_{s}\right)\left(c_{[t+1: T]}\right)\right\}
$$

EXAMPLE. If each one-step time-aggregators is simply the sum of two terms $\left(\Phi_{t}\left\{c_{t}, c_{t+1}\right\}=c_{t}+c_{t+1}\right)$, their composition is just the total sum:

$$
\left(\bigodot_{s=t}^{T-1} \Phi_{s}\right)\left(c_{[t: T]}\right)=\sum_{s=t}^{T} c_{s}
$$

More generally, consider the sequence $\left\{\Phi_{t}\right\}_{t=0}^{T-1}$ of one-step time-aggregators given by

$$
\Phi_{t}\left\{c_{t}, c_{t+1}\right\}=\alpha_{t}\left(c_{t}\right)+\beta_{t}\left(c_{t}\right) c_{t+1}, \forall t \in \llbracket 0, T-1 \rrbracket
$$

where $\left(\alpha_{t}\right)_{t \in \llbracket 0, T-1 \rrbracket}$ and $\left(\beta_{t}\right)_{t \in \llbracket 0, T-1 \rrbracket}$ are sequences of functions, each mapping $\overline{\mathbb{R}}$ into $\mathbb{R}$. With the convention that $\alpha_{T}\left(c_{T}\right)=c_{T}$, we have

$$
\left(\bigodot_{s=t}^{T-1} \Phi_{s}\right)\left\{c_{s}\right\}_{t}^{T}=\sum_{s=t}^{T}\left(\alpha_{s}\left(c_{s}\right) \prod_{r=t}^{s-1} \beta_{r}\left(c_{r}\right)\right), \quad \forall t \in \llbracket 0, T-1 \rrbracket .
$$

\subsubsection{One-Step Uncertainty-Aggregators and their Composition}

Definition 5. Let $t \in \llbracket 0, T \rrbracket$ and $s \in \llbracket t, T \rrbracket$. A $[t: s]$-multiple-step uncertaintyaggregator is a mapping $\mathbb{G}^{[t: s]}$ from $\mathcal{F}\left(\mathbb{W}_{[t: s]} ; \overline{\mathbb{R}}\right)$ into $\overline{\mathbb{R}}$. When $t=s$, we call $\mathbb{G}^{[t: t]}$ a $t$-one-step uncertainty-aggregator. A $[t: s]$-multiple-step uncertaintyaggregator is said to be non-decreasing if it is monotonous with respect to the pointwise partial order of functions. To a $[t: s]$-multiple-step uncertainty-aggregator $\mathbb{G}^{[t: s]}$, we attach a mapping

$$
\mathbb{G}^{[t: s]}: \mathcal{F}\left(\mathbb{W}_{[0: s]} ; \overline{\mathbb{R}}\right) \rightarrow \mathcal{F}\left(\mathbb{W}_{[0: t-1]} ; \overline{\mathbb{R}}\right),
$$

obtained by freezing the first variables (seen as parameters). 
We define the notion of chained sequence of uncertainty-aggregators and their composition as follows.

Definition 6. Let $t \in \llbracket 0, T \rrbracket$ and $s \in \llbracket t+1, T \rrbracket$. Let $\mathbb{G}^{[t: t]}: \mathcal{F}\left(\mathbb{W}_{t} ; \overline{\mathbb{R}}\right) \rightarrow \overline{\mathbb{R}}$ be a $t$-one-step uncertainty-aggregator, and $\mathbb{G}^{[t+1: s]}: \mathcal{F}\left(\mathbb{W}_{[t+1: s]} ; \overline{\mathbb{R}}\right) \rightarrow \overline{\mathbb{R}}$ be a $[t+1: s]$-multiple-step uncertainty-aggregator. We define the $[t: s]$-multiple-step uncertainty-aggregator $\mathbb{G}^{[t: t]} \sqcup \mathbb{G}^{[t: s]}$ by, for all function $A_{t} \in \mathcal{F}\left(\mathbb{W}_{[t: s]} ; \overline{\mathbb{R}}\right)$,

$$
\left(\mathbb{G}^{[t: t]} \sqcup \mathbb{G}^{[t: s]}\right)\left[A_{t}\right]=\mathbb{G}^{[t: t]}\left[w_{t} \mapsto \mathbb{G}^{[t+1: s]}\left[w_{[t+1: s]} \mapsto A_{t}\left(w_{t}, w_{[t+1: s]}\right)\right]\right] .
$$

We say that a sequence $\left\{\mathbb{G}_{t}\right\}_{t=0}^{T}$ of one-step uncertainty-aggregators is a

Definition 8. We inductively define a nested dynamic uncertainty criterion $\left\{\varrho_{t, T}^{N}\right\}_{t=0}^{T}$ by

$$
\begin{aligned}
\varrho_{T}^{N}\left(A_{T}\right) & =\mathbb{G}_{T}\left[A_{T}\right] \\
\varrho_{t, T}^{N}\left(\left\{A_{s}\right\}_{s=t}^{T}\right) & =\mathbb{G}_{t}\left[\Phi_{t}\left\{A_{t}, \varrho_{t+1, T}^{N}\left(\left\{A_{s}\right\}_{s=t+1}^{T}\right)\right\}\right], \forall t \in \llbracket 0, T-1 \rrbracket,
\end{aligned}
$$

for any adapted uncertainty process $\left\{A_{s}\right\}_{s=0}^{T}$.

The following Theorem 9 states that monotonicity is enough to ensure the time-consistency of nested dynamic uncertainty criteria.

Theorem 9. Let $(\Phi, \mathbb{G})$ be a monotonous pair of sequences of aggregators. 205 Then, the nested dynamic uncertainty criterion $\left\{\varrho_{t, T}^{N}\right\}_{t=0}^{T}$ defined by (11) is time-consistent (in the sense of Definition 3). 
Proof. Let $\underline{t}<\bar{t}$ be both in $\llbracket 0, T \rrbracket$. Consider two adapted uncertainty processes $\left\{\underline{A}_{s}\right\}_{0}^{T}$ and $\left\{\bar{A}_{s}\right\}_{0}^{T}$, where $\underline{A}_{s}$ and $\bar{A}_{s}$ maps $\mathbb{W}_{[0: T]}$ into $\overline{\mathbb{R}}$, such that

$$
\begin{array}{rlrl}
\underline{A}_{s} & =\bar{A}_{s}, & \forall s \in \llbracket \underline{t}, \bar{t} \rrbracket, \\
\varrho_{\bar{t}, T}^{N}\left(\left\{\underline{A}_{s}\right\}_{\bar{t}}^{T}\right) & \leq \varrho_{\bar{t}, T}^{N}\left(\left\{\bar{A}_{s}\right\}_{\bar{t}}^{T}\right),
\end{array}
$$

We show by backward induction that, for all $t \in \llbracket \underline{t}, \bar{t} \rrbracket$, the following statement $\left(H_{t}\right)$ holds true:

$$
\left(H_{t}\right) \quad \varrho_{t, T}^{N}\left(\left\{\underline{A}_{s}\right\}_{t}^{T}\right) \leq \varrho_{t, T}^{N}\left(\left\{\bar{A}_{s}\right\}_{t}^{T}\right) .
$$

First, we observe that $\left(H_{\bar{t}}\right)$ holds true by assumption (12b). Second, by $\left(H_{t}\right)$, and as $\underline{A}_{t-1}=\bar{A}_{t-1}$ monotonicity of $\Phi_{t-1}$ yields

$$
\Phi_{t-1}\left\{\underline{A}_{t-1}, \varrho_{t, T}^{N}\left(\left\{\underline{A}_{s}\right\}_{t}^{T}\right)\right\} \leq \Phi_{t-1}\left\{\bar{A}_{t-1}, \varrho_{t, T}^{N}\left(\left\{\bar{A}_{s}\right\}_{t}^{T}\right)\right\} .
$$

Monotonicity of $\mathbb{G}_{t-1}$ then gives

$$
\mathbb{G}_{t-1}\left[\Phi_{t-1}\left\{\underline{A}_{t-1}, \varrho_{t, T}^{N}\left(\left\{\underline{A}_{s}\right\}_{t}^{T}\right)\right\}\right] \leq \mathbb{G}_{t-1}\left[\Phi_{t-1}\left\{\bar{A}_{t-1}, \varrho_{t, T}^{N}\left(\left\{\bar{A}_{s}\right\}_{t}^{T}\right)\right\}\right] .
$$

By definition of $\varrho_{t-1, T}^{N}$ in (11), we obtain $\left(H_{t-1}\right)$.

Remark 10. In Definition 8, we build nested aggregators, first starting by aggregating with respect to time, second with respect to uncertainty. If we aggregate first with respect to uncertainty, second with respect to time, we obtain the dynamic uncertainty criterion given by

$$
\begin{aligned}
\varrho_{T}^{N^{\prime}}\left(A_{T}\right) & =\mathbb{G}_{T}\left[A_{T}\right], \\
\varrho_{t, T}^{N^{\prime}}\left(\left\{A_{s}\right\}_{s=t}^{T}\right) & =\Phi_{t}\left\{\mathbb{G}_{t}\left[A_{t}\right], \mathbb{G}_{t}\left[\varrho_{t+1, T}^{N^{\prime}}\left(\left\{A_{s}\right\}_{s=t+1}^{T}\right)\right]\right\}, \forall t \in \llbracket 0, T-1 \rrbracket .
\end{aligned}
$$

It is shown in [16, Chapter 2] that monotonicity is also sufficient to yield timeconsistency.

\subsection{Time-Consistency for Block-Aggregated Criteria}

Nested uncertainty criteria carry the time-consistency property in the very manner they are built. However, nested uncertainty criterion are not the most natural candidates to assess risk and their economic interpretation is delicate. In practice, uncertainty criterion are more often given in block-aggregation form - integrate with respect to all times, then with respect to all uncertainties than in nested form.

We first propose a commutation property that will allow to go from one formulation to the other, and that will stand as one of the key ingredients for a DPE and lead to the time-consistency result in Theorem 14. 
Definition 11. Let $t \in \llbracket 0, T \rrbracket$ and $s \in \llbracket t+1, T \rrbracket$. A $[t: s]$-multiple-step uncertaintyaggregator $\mathbb{G}^{[t: s]}$ is said to commute with a one-step time-aggregator $\Phi$ if

$$
\mathbb{G}^{[t: s]}\left[w_{[t: s]} \mapsto \Phi\left\{c, D_{t}\left(w_{[t: s]}\right)\right\}\right]=\Phi\left\{c, \mathbb{G}^{[t: s]}\left[w_{[t: s]} \mapsto D_{t}\left(w_{[t: s]}\right)\right]\right\},
$$

for any function $D_{t} \in \mathcal{F}\left(\mathbb{W}_{[t: s]} ; \overline{\mathbb{R}}\right)$ and any extended scalar $c \in \overline{\mathbb{R}}$.

We say that a $(\Phi, \mathbb{G})$ is a commuting pair of sequence of aggregators if $\mathbb{G}_{t}$ commutes with $\Phi_{s}$, for any $0 \leq s<t \leq T$.

EXAMPLE. If $\left(\mathbb{W}_{t}, \mathcal{F}_{t}, \mathbb{P}_{t}\right)$ is a probability space and if

$$
\Phi\left\{c, c_{t}\right\}=\alpha(c)+\beta(c) c_{t},
$$

where $\alpha: \overline{\mathbb{R}} \rightarrow \mathbb{R}$ and $\beta: \overline{\mathbb{R}} \rightarrow \mathbb{R}_{+}$, then the extended ${ }^{2}$ expectation $\mathbb{G}^{[t: t]}=\mathbb{E}_{\mathbb{P}_{t}}$ commutes with $\Phi$.

The following lemma shows how commutation of time-step aggregators leads to commutation of multi-step aggregators.

Lemma 12. Consider a commuting pair $(\Phi, \mathbb{G})$ of aggregators. Then, $\stackrel{\leftrightarrow}{s=t}_{\vec{G}}^{T} \mathbb{G}_{s}$ commutes with $\Phi_{r}$, for any $0 \leq r<t \leq T$, that is,

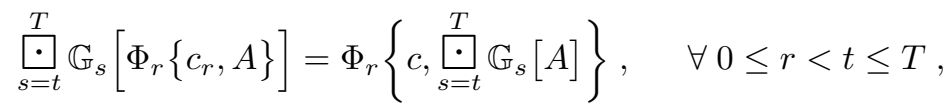

for any extended scalar $c \in \overline{\mathbb{R}}$ and any function $A \in \mathcal{F}\left(\mathbb{W}_{[0: T]} ; \overline{\mathbb{R}}\right)$.

Beware that, for a given time $t$, we require the commutation of all subsequent uncertainty-aggregators $\mathbb{G}_{t+1}, \ldots, \mathbb{G}_{T}$ with the time aggregator $\Phi_{t}$ (see Definition 11). The proof of Lemma 12 by induction, is detailed in Appendix B.1

\subsubsection{Time-Consistency for Block-Aggregated Criteria}

We now define block dynamic uncertainty criteria and prove their timeconsistency under a commutation property.

Definition 13. We define the block dynamic uncertainty criterion $\left\{\varrho_{t, T}^{B}\right\}_{t=0}^{T}$ by

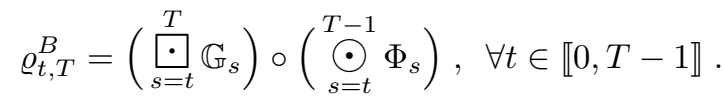

The following Theorem 14 is our main result on time-consistency in the block-aggregation case.

\footnotetext{
${ }^{2}$ We set $\beta \geq 0$, so that, when $C_{t} \in \mathcal{F}\left(\mathbb{W}_{t} ; \overline{\mathbb{R}}\right)$ is not integrable with respect to $\mathbb{P}_{t}$, the equality (14) still holds true.
} 
Theorem 14. Let $(\Phi, \mathbb{G})$ be a commuting and monotonous pair of sequences of aggregators. Then the block dynamic uncertainty criterion $\left\{\varrho_{t, T}^{B}\right\}_{t=0}^{T}$ defined by (17) is time-consistent.

Proof. Since, for any $0 \leq s<t \leq T$, $\mathbb{G}_{t}$ commutes with $\Phi_{s}$, the blockdynamic uncertainty criterion $\left\{\varrho_{t, T}^{B}\right\}_{t=0}^{T}$, given by Definition 17, coincides with $\left\{\varrho_{t, T}^{N}\right\}_{t=0}^{T}$, given by Definition 8 . Indeed, we prove that $\left\{\varrho_{t, T}^{B}\right\}_{t=0}^{T}$ satisfies the backward induction (11).

With the convention that $\left(\bigodot_{r=T}^{T-1} \Phi_{r}\right)$ is the identity mapping, we have $\varrho_{T}^{B}=$ $\mathbb{G}_{T}$, that is, 11a.

Then, let $t$ be fixed. For any $\left\{A_{s}\right\}_{t}^{T} \in\left[\mathcal{F}\left(\mathbb{W}_{[0: s]} ; \overline{\mathbb{R}}\right)\right]_{s=t}^{T}$, we have:

$$
\begin{aligned}
& \varrho_{t}^{B}\left(\left\{A_{s}\right\}_{s=t}^{T}\right)=\stackrel{\bullet}{r=t}_{s}^{s} \mathbb{G}_{r}\left[\bigodot_{r=t}^{T-1} \Phi_{r}\left\{\left\{A_{s}\right\}_{s=t}^{T}\right\}\right] \text { by (17), } \\
& =\mathbb{G}_{t}\left[\stackrel{s}{\stackrel{\bullet}{\cdot}} \mathbb{G}_{r}\left[\bigodot_{r=t}^{T-1} \Phi_{r}\left\{\left\{A_{s}\right\}_{s=t}^{T}\right\}\right]\right] \text { by } 100, \\
& =\mathbb{G}_{t}\left[\underset{r=t+1}{\stackrel{s}{\ominus}} \mathbb{G}_{r}\left[\Phi_{t}\left\{A_{t},\left(\bigodot_{r=t+1}^{T-1} \Phi_{r}\right)\left\{A_{s}\right\}_{s=t+1}^{T}\right\}\right]\right] \text { by (5), } \\
& =\mathbb{G}_{t}\left[\Phi_{t}\left\{A_{t}, \underset{r=t+1}{\stackrel{s}{\cdot}} \mathbb{G}_{r}\left[\left(\bigodot_{r=t+1}^{T-1} \Phi_{r}\right)\left\{A_{s}\right\}_{s=t+1}^{T}\right]\right\}\right] \text { by (16) } \\
& =\mathbb{G}_{t}\left[\Phi_{t}\left(A_{t}, \varrho_{t+1}^{B}\left(\left\{A_{s}\right\}_{s=t+1}^{T}\right)\right)\right] \text { by (17). }
\end{aligned}
$$

Thus, $\left\{\varrho_{t, T}^{B}\right\}_{t=0}^{T}$ satisfies the backward induction (11).

Remark 15. In Definition 13, we build block-aggregators, first starting by aggregating with respect to time, second with respect to uncertainty. If we aggregate first with respect to uncertainty, second with respect to time, we obtain another dynamic uncertainty criterion. However, to show time-consistency would require monotonicity and another notion of commutation, less widespread than Definition 11. See [16, Chapter 2] for more details on the subject.

In [16. Chapter 2], the construction of this $\$ 2$ is extended to Markovian aggregators. Roughly speaking, the time-step time and uncertainty aggregators are parametrized by a state, that follows a control dynamical system. All results remain true if the commutation property holds true for every possible value of the underlying state.

\section{Building up Time-Consistent Intertemporal Optimization Prob- lems}

In $\$ 2$ we considered dynamic uncertainty criterion that value (the risk of) a stream of costs. We now use such criteria to formulate intertemporal opti- 
mization problems under uncertainty. In 3.1 , we lay out the basic material to formulate intertemporal optimization problems. Then, we provide definition of

time-consistency in $\$ 3.2$, and time-consistency results in $\$ 3.3$

\subsection{Ingredients for Optimal Control Problems}

In $\$ 3.1 .1$, we recall the formalism of Control Theory, with dynamical system, state, control and costs. In 3.1 .2 , we show how to produce adapted uncertainty processes of costs, that will be the inputs to dynamic uncertainty criteria.

\subsubsection{Dynamical System, State, Control and Costs}

Let $T \geq 2$ be an integer. We define a control $T$-stage dynamical system as follows. We consider sequences of sets of states $\left(\left\{\mathbb{X}_{t}\right\}_{0}^{T}\right)$, controls $\left(\left\{\mathbb{U}_{t}\right\}_{0}^{T-1}\right)$, and uncertainties $\left(\left\{\mathbb{W}_{t}\right\}_{0}^{T}\right)$. We also consider sequences of functions representing dynamics $\left(\left\{f_{t}\right\}_{0}^{T-1}\right.$, where $\left.f_{t}: \mathbb{X}_{t} \times \mathbb{U}_{t} \times \mathbb{W}_{t} \rightarrow \mathbb{X}_{t+1}\right)$ constraints $\left(\left\{U_{t}\right\}_{0}^{T-1}\right.$, where $U_{t}: \mathbb{X}_{t} \rightrightarrows \mathbb{U}_{t}$ is a set-valued function), and costs $\left(\left\{J_{t}\right\}_{0}^{T-1}\right.$ with $J_{t}: \mathbb{X}_{t} \times \mathbb{U}_{t} \times \mathbb{W}_{t} \mapsto \overline{\mathbb{R}}$ being instantaneous cost functions $)$ and $J_{T}: \mathbb{X}_{T} \times \mathbb{W}_{T} \rightarrow \overline{\mathbb{R}}$ the final cost function ${ }^{3}$

A policy $\pi=\left(\pi_{t}\right)_{0}^{T-1}$ is a sequence of functions $\pi_{t}: \mathbb{X}_{t} \rightarrow \mathbb{U}_{t}$, and we denote by $\Pi$ the set of all policies. More generally, for all $t \in \llbracket 0, T \rrbracket$, we call (tail) policy a sequence $\pi=\left(\pi_{s}\right)_{t}^{T-1}$ and we denote by $\Pi_{t}$ the set of all such policies.

We restrict our search of optimal solutions to so-called admissible policies belonging to a subset $\Pi^{\text {ad }} \subset \Pi$. An admissible policy $\pi \in \Pi^{\text {ad }}$ always satisfies:

$$
\pi_{t}(x) \in U_{t}(x), \quad \forall x \in \mathbb{X}_{t}, \quad \forall t \in \llbracket 0, T-1 \rrbracket .
$$

We can express in $\Pi^{\text {ad }}$ other types of constraints, such as measurability or integrability ones when we are in a stochastic setting. Naturally, we set $\Pi_{t}^{a d}=$ $\Pi_{t} \cap \Pi^{a d}$.

Definition 16. For any time $t \in \llbracket 0, T \rrbracket$, state $x \in \mathbb{X}_{t}$ and policy $\pi \in \Pi$, the flow $\left\{X_{t, s}^{x, \pi}\right\}_{s=t}^{T}$ of the dynamics $\left\{f_{t}\right\}_{0}^{T-1}$ is defined by the forward induction:

$$
\forall w \in \mathbb{W}_{[0: T]},\left\{\begin{aligned}
X_{t, t}^{x, \pi}(w) & =x, \\
X_{t, s+1}^{x, \pi}(w) & =f_{s}\left(X_{t, s}^{x, \pi}(w), \pi_{s}\left(X_{t, s}^{x, \pi}(w)\right), w_{s}\right), \forall s \in \llbracket t, T \rrbracket .
\end{aligned}\right.
$$

The expression $X_{t, s}^{x, \pi}(w)$ is the state $x_{s} \in \mathbb{X}_{s}$ reached at time $s \in \llbracket 0, T \rrbracket$, when starting at time $t \in \llbracket 0, s \rrbracket$ from state $x \in \mathbb{X}_{t}$ and following the dynamics $\left\{f_{r}\right\}_{0}^{T-1}$ with the policy $\pi \in \Pi$ along the scenario $w \in \mathbb{W}_{[0: T]}$.

\footnotetext{
${ }^{3}$ For notational consistency with the $J_{t}$ for $t=\llbracket 0, T-1 \rrbracket$, we will often write $J_{T}(x, u, w)$ to mean $J_{T}(x, w)$.
} 
Remark 17. For $0 \leq t \leq s \leq T$, the flow $X_{t, s}^{x, \pi}$ is a function that maps the set $\mathbb{W}_{[0: T]}$ of scenarios into the state space $\mathbb{X}_{s}$ :

$$
X_{t, s}^{x, \pi}: \mathbb{W}_{[0: T]} \rightarrow \mathbb{X}_{s}
$$

By (18),

- when $t>0$, the expression $X_{t, s}^{x, \pi}(w)$ depends only on the inner part $w_{[t: s-1]}$ of the scenario $w=w_{[0: T]}$, hence depends neither on the head $w_{[0: t-1]}$, nor on the tail $w_{[s: T]}$,

- when $t=0$, the expression $X_{0, s}^{x, \pi}(w)$ in $(18)$ depends only on the head $w_{[0: s-1]}$ of the scenario $w=w_{[0: T]}$, hence does not depend on the tail $w_{[s: T]}$.

This is why we often consider that the flow $X_{t, s}^{x, \pi}$ is a function that maps the set $\mathbb{W}_{[t: s-1]}$ of scenarios into the state space $\mathbb{X}_{s}$ :

$$
X_{t, s}^{x, \pi}: \mathbb{W}_{[t: s-1]} \rightarrow \mathbb{X}_{s}, \quad \forall s \in \llbracket 1, T \rrbracket, \quad \forall t \in \llbracket 0, s-1 \rrbracket .
$$

A state trajectory is a realization of the flow $\left\{X_{0, s}^{x, \pi}(w)\right\}_{s=0}^{T}$ for a given scenario $w \in \mathbb{W}_{[0: T]}$. The flow property

$$
X_{t, s}^{x, \pi} \equiv X_{s^{\prime}, s}^{X_{t, s}^{x, \pi}, \pi}, \forall t, s, s^{\prime}, t<s^{\prime}<s, \forall x \in \mathbb{X}_{t}
$$

expresses the fact that we can stop anywhere along a state trajectory and start again.

\subsubsection{Producing Streams of Costs}

With a policy and a scenario, we obtain state and control trajectories that we plug into the instantaneous cost functions $J_{s}$ to deliver streams of costs.

Definition 18. For a given policy $\pi \in \Pi$, and for all times $t \in \llbracket 0, T \rrbracket$ and $s \in \llbracket t, T \rrbracket$, we define the uncertain costs evaluated along the state trajectories by:

$$
J_{t, s}^{x, \pi}: \quad w \in \mathbb{W}_{[0: T]} \longmapsto J_{s}\left(X_{t, s}^{x, \pi}(w), \pi\left(X_{t, s}^{x, \pi}(w)\right), w_{s}\right)
$$

Remark 19. By Remark 17 , we consider that $J_{t, s}^{x, \pi}$ is a function that maps the set $\mathbb{W}_{[t: s]}$ of scenarios into $\mathbb{R}$ :

$$
J_{t, s}^{x, \pi}: \quad \mathbb{W}_{[t: s]} \rightarrow \overline{\mathbb{R}}, \forall s \in \llbracket 0, T \rrbracket, \forall t \in \llbracket 0, s \rrbracket .
$$

As a consequence, the stream $\left\{J_{0, s}^{x, \pi}\right\}_{s=0}^{T}$ of costs is an adapted uncertainty process (see Definition 1). 
By (22) and (18), we have, for all $t \in \llbracket 0, T \rrbracket$ and $s \in \llbracket t+1, T \rrbracket$,

$$
\forall w_{[t: T]} \in \mathbb{W}_{[t: T]},\left\{\begin{aligned}
J_{t, t}^{x, \pi}\left(w_{t}\right) & =J_{t}\left(x, \pi_{t}(x), w_{t}\right), \\
J_{t, s}^{x, \pi}\left(w_{t},\left\{w_{r}\right\}_{t+1}^{T}\right) & =J_{t+1, s}^{f_{t}\left(x, \pi_{t}(x), w_{t}\right), \pi}\left(\left\{w_{r}\right\}_{t+1}^{T}\right) .
\end{aligned}\right.
$$

We relate dynamic uncertainty criteria and optimization problems as follows.

Definition 20. Given a Markov dynamic uncertainty criterion $\left\{\left\{\varrho_{t, T}^{x_{t}}\right\}_{x_{t} \in \mathbb{X}_{t}}\right\}_{t=0}^{T}$, we define a Markov optimization problem as the following sequence of families of optimization problems, indexed by $t \in \llbracket 0, T \rrbracket$, and $x \in \mathbb{X}_{t}$ :

$$
\left(\mathfrak{P}_{t}\right)(x) \min _{\pi \in \Pi^{\text {ad }}} \varrho_{t, T}^{x}\left(\left\{J_{t, s}^{x, \pi}\right\}_{s=t}^{T}\right) .
$$

305 Each Problem 25 is indeed well defined by $1 \mathrm{bb}$, because $\left\{J_{t, s}^{x, \pi}\right\}_{s=t}^{T}$ belongs to $\left[\mathcal{F}\left(\mathbb{W}_{[t: s]} ; \overline{\mathbb{R}}\right)\right]_{s=t}^{T}$ by 23 .

\subsection{Definition of Time-Consistency for Markov Optimization Problems}

With the formalism of $\$ 2.1$, we can now give a definition of time-consistency for Markov optimization problems.

For the clarity of exposition, suppose for a moment that any optimization problem $\left(\mathfrak{P}_{t}\right)(x)$ in 25 has a unique solution, a policy that we denote $\pi^{t, x}=$ $\left\{\pi_{s}{ }^{t, x}\right\}_{s=t}^{T-1} \in \Pi_{t}^{\text {ad }}$ to stress that it parametrically depends on $t, x$ as $\left(\mathfrak{P}_{t}\right)(x)$ does. Consider $0 \leq \underline{t}<\bar{t} \leq T$. Suppose that, starting from the state $\underline{x}$ at time $\underline{t}$, the flow 18 drives you to

$$
\bar{x}=X_{\underline{t}, \bar{t}}^{\underline{x}, \pi}(w), \pi=\pi^{\underline{t}, \underline{x}}
$$

at time $\bar{t}$, along the scenario $w \in \mathbb{W}_{[0: T]}$ and adopting the optimal policy $\pi^{\underline{t}}, \underline{x} \in$ $\Pi_{\underline{t}}^{\text {ad }}$. Arrived at $\bar{x}$, you solve $\left(\mathfrak{P}_{\bar{t}}\right)(\bar{x})$ and get the optimal policy $\pi^{\bar{t}, \bar{x}}=\left\{\pi_{s}^{\bar{t}, \bar{x}}\right\}_{s=\bar{t}}^{T-1} \in$ $\Pi_{\bar{t}}^{\underline{a}}$. Time-consistency holds true when

$$
\pi_{s}{ }^{\bar{t}, \bar{x}}=\pi_{s}^{\underline{t}, \underline{x}}, \forall s \geq \bar{t},
$$
after time $\bar{t}$, with the "old" optimal policy, obtained by solving $\left(\mathfrak{P}_{t}\right)(x)$. In other words, you "stick to your plans" (here, a plan is a policy) and do not reconsider your policy whenever you stop along an optimal path and optimize ahead from this stop point.

To account for non-uniqueness of optimal policies, we propose the following formal definition. 
Definition 21. We say that the Markov optimization problem 25) of Definition 20 is time-consistent if, for any couple of times $\underline{t} \leq \bar{t}$ in $\llbracket 0, T \rrbracket$ and any state $\underline{x} \in \mathbb{X}_{\underline{t}}$, the following property holds: there exists a policy $\pi^{\sharp}=\left\{\pi_{s}^{\sharp}\right\}_{s=t}^{T-1} \in$ $\Pi_{\underline{t}}^{\text {ad }}$ such that

- $\left\{\pi_{s}^{\sharp}\right\}_{s=\underline{t}}^{T-1}$ is optimal for Problem $\mathfrak{P}_{\underline{t}}(\underline{x})$;

- the tail policy $\left\{\pi_{s}^{\sharp}\right\}_{s=\bar{t}}^{T-1}$ is optimal for Problem $\mathfrak{P}_{\bar{t}}(\bar{x})$, where $\bar{x} \in \mathbb{X}_{\bar{t}}$ is any state achieved by the flow $X_{\underline{\underline{t}}, \bar{t}}^{\underline{x}, \pi^{\sharp}}$ in 18$)$.

We stress that the above definition of time-consistency of a sequence of 325 families of optimization problems is contingent on the state $x$ and on the dynamics $\left\{f_{t}\right\}_{0}^{T-1}$ by the flow (18). In particular, we assume that, at each time step, the control is taken only in function of the state: this defines the class of solutions as policies that are feedbacks of the state $x$ (such restriction is justified in the Markovian case, for example).

\subsection{Time-Consistency for Optimal Control Problems}

We now provide time-consistency results, differing whether in the nestedaggregation or in the block-aggregation case.

\subsubsection{Time-Consistency for Nested Criteria}

We define the nested Markov optimization problem formally by

$$
\left(\mathfrak{P}_{t}^{N}\right)(x) \quad V_{t}^{N}(x)=\min _{\pi \in \Pi_{t}^{\text {ad }}} \varrho_{t, T}^{N}\left(\left\{J_{t, s}^{x, \pi}\right\}_{s=t}^{T}\right), \forall t \in \llbracket 0, T \rrbracket, \forall x \in \mathbb{X}_{t},
$$

where the functions $J_{t, s}^{x, \pi}$ are defined by 22 , and the nested uncertainty crite335 rion $\left\{\varrho_{t, T}^{N}\right\}_{t=0}^{T}$ by 11 .

The following Proposition 22 expresses sufficient conditions under which any Problem $\left(\mathfrak{P}_{t}^{N}\right)(x)$, for any time $t \in \llbracket 0, T-1 \rrbracket$ and any state $x \in \mathbb{X}_{t}$, can be solved by means of a Dynamic Programming Equation (DPE).

Proposition 22. Let $(\Phi, \mathbb{G})$ be a monotonous pair of sequences of aggregators. Assume that there exists an admissible policy $\pi^{\sharp} \in \Pi^{\text {ad }}$ such that

$$
\begin{array}{r}
\pi_{t}^{\sharp}(x) \in \underset{u \in U_{t}(x)}{\arg \min } \mathbb{G}_{t}\left[\Phi_{t}\left\{J_{t}(x, u, \cdot), V_{t+1}^{N} \circ f_{t}(x, u, \cdot)\right\}\right], \\
\forall t \in \llbracket 0, T-1 \rrbracket, \forall x \in \mathbb{X}_{t} .
\end{array}
$$

Then, $\pi^{\sharp}$ is an optimal policy for any Problem $\left(\mathfrak{P}_{t}^{N}\right)(x)$, for all $t \in \llbracket 0, T \rrbracket$ and for all $x \in \mathbb{X}_{t}$, and the value functions $V_{t}$ satisfy the DPE

$$
\begin{aligned}
V_{T}^{N}(x) & =\mathbb{G}_{T}\left[J_{T}(x, \cdot)\right], \quad \forall x \in \mathbb{X}_{T}, \\
V_{t}^{N}(x) & =\min _{u \in U_{t}(x)} \mathbb{G}_{t}\left[\Phi_{t}\left\{J_{t}(x, u, \cdot), V_{t+1}^{N} \circ f_{t}(x, u, \cdot)\right\}\right], \\
& \forall t \in \llbracket 0, T-1 \rrbracket, \forall x \in \mathbb{X}_{t} .
\end{aligned}
$$


Remark 23. It may be difficult to prove the existence of a measurable selection among the solutions of (29). Since it is not our intent to consider such issues, we make the assumption that an admissible policy $\pi^{\sharp} \in \Pi^{\mathrm{ad}}$ exists, where the definition of the set $\Pi^{\text {ad }}$ is supposed to include all proper measurability conditions.

The proof of Proposition 22 is detailed in the appendix.

The following Theorem 9 is our main result on time-consistency in the nested-aggregation case.

Theorem 24. Let $(\Phi, \mathbb{G})$ be a monotonous pair of sequences of aggregators. Then

1. the nested dynamic uncertainty criterion $\left\{\varrho_{t, T}^{N}\right\}_{t=0}^{T}$ defined by (11) is timeconsistent;

2. the Markov optimization problem $\left\{\left\{\left(\mathfrak{P}_{t}^{N}\right)(x)\right\}_{x \in \mathbb{X}_{t}}\right\}_{t=0}^{T}$ defined in 28] is time-consistent, as soon as there exists an admissible policy $\pi^{\sharp} \in \Pi^{\text {ad }}$ such that (29) holds true.

\subsubsection{Time-Consistency for Block-Aggregated Criteria}

We define the block Markov optimization problem formally by

$$
\left(\mathfrak{P}_{t}^{B}\right)(x) \quad \min _{\pi \in \Pi_{t}^{\text {ad }}} \varrho_{t, T}^{B}\left(\left\{J_{t, s}^{x, \pi}\right\}_{s=t}^{T}\right), \forall t \in \llbracket 0, T \rrbracket, \forall x \in \mathbb{X}_{t},
$$

where the functions $J_{t, s}^{x, \pi}$ are defined by (22), and the block uncertainty criterion $\left\{\varrho_{t, T}^{B}\right\}_{t=0}^{T}$ by 17 .

The following Theorem 25 is our main result on time-consistency in the block-aggregation case. We do not give the proof since it directly follows from 360 Theorem 9 and Theorem 14

Theorem 25. Let $(\Phi, \mathbb{G})$ be a monotonous and commuting pair of sequences of aggregators. Then

1. the block-aggregated dynamic uncertainty criterion $\left\{\varrho_{t, T}^{B}\right\}_{t=0}^{T}$ defined by (17) is time-consistent;

2. the Markov optimization problem $\left\{\left\{\left(\mathfrak{P}_{t}^{B}\right)(x)\right\}_{x \in \mathbb{X}_{t}}\right\}_{t=0}^{T}$ defined in (31) is time-consistent, as soon as there exists an admissible policy $\pi^{\sharp} \in \Pi^{\text {ad }}$ such that (29) holds true.

Remark 26. Theorem 24 and 25 are given for criteria obtained via time followed by uncertainty aggregation. When uncertainty precedes time aggregation, we can obtain similar results, at the price of a different definition of commuting aggregators [16, Chapter 2].

In [16. Chapter 2], the time consitency result is extended to Markovian aggregators, made of one-step time and uncertainty aggregators parameterized by the state. All results remain true if the commutation property holds true for 


\section{Applications}

We end by providing classes of dynamic uncertainty criteria and corresponding intertemporal optimization problems that display time-consistency, as well as examples of applications.

\subsection{Coherent Risk Measures}

We introduce a class of dynamic uncertainty criteria, that are related to coherent risk measures, and we show that they display time-consistency. We thus extend, to more general one-step time-aggregators, results known for the sum (see e.g. [17, 18]).

We denote by $\mathcal{P}\left(\mathbb{W}_{t}\right)$ the set of probabilities over the set $\mathbb{W}_{t}$ endowed with the $\sigma$-algebra $\mathcal{W}_{t}$. Let $\mathcal{P}_{0} \subset \mathcal{P}\left(\mathbb{W}_{0}\right), \ldots, \mathcal{P}_{T} \subset \mathcal{P}\left(\mathbb{W}_{T}\right)$. If $A$ and $B$ are sets of probabilities, then $A \otimes B$ is defined as

$$
A \otimes B=\left\{\mathbb{P}_{A} \otimes \mathbb{P}_{B} \mid \mathbb{P}_{A} \in A, \mathbb{P}_{B} \in B\right\} .
$$

Let $\left(\alpha_{t}\right)_{t \in \llbracket 0, T-1 \rrbracket}$ and $\left(\beta_{t}\right)_{t \in \llbracket 0, T-1 \rrbracket}$ be sequences of functions, each mapping $\overline{\mathbb{R}}$ into $\mathbb{R}$, with the additional property that $\beta_{t} \geq 0$, for all $t \in \llbracket 0, T-1 \rrbracket$. We set, for all $t \in \llbracket 0, T \rrbracket$,

$$
\varrho_{t, T}^{\text {co }}\left(\left\{A_{s}\right\}_{t}^{T}\right)=\sup _{\mathbb{P}_{t} \in \mathcal{P}_{t}} \mathbb{E}_{\mathbb{P}_{t}}\left[\cdots \sup _{\mathbb{P}_{T} \in \mathcal{P}_{T}} \mathbb{E}_{\mathbb{P}_{T}}\left[\sum_{s=t}^{T}\left(\alpha_{s}\left(A_{s}\right) \prod_{r=t}^{s-1} \beta_{r}\left(A_{r}\right)\right)\right] \cdots\right],
$$

385 for any adapted uncertain process $\left\{A_{t}\right\}_{0}^{T}$, with the convention that $\alpha_{T}\left(c_{T}\right)=$ $c_{T}$.

Proposition 27. Time-consistency holds true for

- the dynamic uncertainty criterion $\left\{\varrho_{t, T}^{c o}\right\}_{t=0}^{T}$ given by [33,

- the Markov optimization problem

$$
\min _{\pi \in \Pi^{\text {ad }}} \varrho_{t, T}^{c o}\left(\left\{J_{t, s}^{x, \pi}\right\}_{s=t}^{T}\right), \forall t \in \llbracket 0, T \rrbracket, \forall x \in \mathbb{X}_{t},
$$

where $J_{t, s}^{x, \pi}(w)$ is defined by 22 , as soon as there exists an admissible policy $\pi^{\sharp} \in \Pi^{\text {ad }}$ such that, for all $t \in \llbracket 0, T-1 \rrbracket$, for all $x \in \mathbb{X}_{t}$,

$\pi_{t}^{\sharp}(x) \in \underset{u \in U_{t}(x)}{\arg \min } \sup _{\mathbb{P}_{t} \in \mathcal{P}_{t}}\left\{\mathbb{E}_{\mathbb{P}_{t}}\left[\alpha_{t}\left(J_{t}(x, u, \cdot)\right)+\beta_{t}\left(J_{t}(x, u, \cdot)\right) V_{t+1} \circ f_{t}(x, u, \cdot)\right]\right\}$,

where the value functions are given by the following DPE

$$
\begin{aligned}
V_{T}(x)= & \sup _{\mathbb{P}_{T} \in \mathcal{P}_{T}} \mathbb{E}_{\mathbb{P}_{T}}\left[J_{T}(x, \cdot)\right] \\
V_{t}(x)=\min _{u \in U_{t}(x)} \sup _{\mathbb{P}_{t} \in \mathcal{P}_{t}}\left\{\mathbb{E}_{\mathbb{P}_{t}}[\right. & \alpha_{t}\left(J_{t}(x, u, \cdot)\right) \\
& \left.\left.\quad+\beta_{t}\left(J_{t}(x, u, \cdot)\right) V_{t+1} \circ f_{t}(x, u, \cdot)\right]\right\} .
\end{aligned}
$$


Proof. The setting is that of Theorem 14 and Proposition 22, where the onestep time-aggregators are defined by

$$
\Phi_{t}\left\{c_{t}, c_{t+1}\right\}=\alpha_{t}\left(c_{t}\right)+\beta_{t}\left(c_{t}\right) c_{t+1}, \quad \forall t \in \llbracket 0, T-1 \rrbracket, \forall\left(c_{t}, c_{t+1}\right) \in \overline{\mathbb{R}}^{2},
$$

and the one-step uncertainty-aggregators are defined by

$$
\mathbb{G}_{t}\left[C_{t}\right]=\sup _{\mathbb{P}_{t} \in \mathcal{P}_{t}} \mathbb{E}_{\mathbb{P}_{t}}\left[C_{t}\right], \quad \forall t \in \llbracket 0, T-1 \rrbracket, \forall C_{t} \in \mathcal{F}\left(\mathbb{W}_{t} ; \overline{\mathbb{R}}\right) .
$$

The DPE (35) is the DPE (30), which holds true as soon as the assumptions of Theorem 14 hold true (mainly that we have a commuting monotonous pair of operators).

First, we prove that, for any $0 \leq t<s \leq T, \mathbb{G}_{s}$ commutes with $\Phi_{t}$ (this is a special case of Proposition 30 shown in appendix). Indeed, letting $c_{t}$ be an extended real number in $\overline{\mathbb{R}}$ and $C_{s}$ a function in $\mathcal{F}\left(\mathbb{W}_{s} ; \overline{\mathbb{R}}\right)$, we have

$$
\begin{aligned}
\mathbb{G}_{s}\left[\Phi_{t}\left\{c_{t}, C_{s}\right\}\right] & =\sup _{\mathbb{P}_{s} \in \mathcal{P}_{s}}\left\{\mathbb{E}_{\mathbb{P}_{s}}\left[\alpha\left(c_{t}\right)+\beta\left(c_{t}\right) C_{s}\right]\right\} & & \text { by } 36 \mathrm{~b} \text { and } 36 \mathrm{a}) \\
& =\alpha_{t}\left(c_{t}\right)+\beta_{t}\left(c_{t}\right) \sup _{\mathbb{P}_{s} \in \mathcal{P}_{s}}\left\{\mathbb{E}_{\mathbb{P}_{s}}\left[C_{s}\right]\right\} & & \text { as } \beta_{t} \geq 0, \\
& =\alpha_{t}\left(c_{t}\right)+\beta_{t}\left(c_{t}\right) \mathbb{G}_{s}\left[C_{s}\right] & & \text { by 36b), } \\
& =\Phi_{t}\left\{c_{t}, \mathbb{G}_{s}\left[C_{s}\right]\right\} & & \text { by 36a. }
\end{aligned}
$$

Second, we observe that $\mathbb{G}_{t}$ is non-decreasing (see Definition 5), and that $c_{t+1} \in$ $\overline{\mathbb{R}} \mapsto \Phi_{t}\left\{c_{t}, c_{t+1}\right\}=\alpha_{t}\left(c_{t}\right)+\beta_{t}\left(c_{t}\right) c_{t+1}$ is non-decreasing, for any $c_{t} \in \overline{\mathbb{R}}$.

The one-step uncertainty-aggregators $\mathbb{G}_{t}$ in $(36 \mathrm{~b})$ correspond to a coherent risk measure (see [19]). In fact, our result extends to aggregators of the form $\theta \sup _{\mathbb{P} \in \mathcal{P}} \mathbb{E}_{\mathbb{P}}+(1-\theta) \inf _{\mathbb{Q} \in \mathcal{Q}} \mathbb{E}_{\mathbb{Q}}$, with $\theta \in[0,1]$, and even to more complex convex combinations of infima and suprema as shown in Proposition 31 in Appendix A.

Our result extends to a Markovian setting where one-step aggregators $\mathbb{G}_{t}$ and $\Phi_{t}$ are indexed by the state $x$. More precisely, the coefficient $\alpha_{t}$ and $\beta_{t}$ and the set of probability $\mathcal{P}_{t}$ can depend on the state $x$.

Our result differs from the work of A. Ruszczyński [17, Theorem 2] in two ways. On the one hand, Ruszczyński provides arguments to show that there exists an optimal Markovian policy among the set of adapted policies (that is, having a policy taking as argument the whole past uncertainties would not give a better cost than a policy taking as argument the current value of the state). We do not tackle this issue since we directly deal with policies as functions of the state. Where we suppose that there exists an admissible policy $\pi^{\sharp} \in \Pi^{\text {ad }}$ such that (29) holds true, Ruszczyński gives conditions ensuring this property. On the 410 other hand, where Ruszczyński restricts to the sum to aggregate instantaneous costs, we consider more general one-step time-aggregators $\Phi_{t}$. For instance, our results applies to the product of costs. 


\subsection{Convex Risk Measures}

We introduce a class of dynamic uncertainty criteria, that are related to ${ }_{415}$ convex risk measures (see [20]), and we show that they display time-consistency. We consider the same setting as in 4.1 , with the restriction that $\beta_{t} \equiv 1$ in 33 . and an additional data $\left(\Upsilon_{t}\right)_{t \in \llbracket 0, T \rrbracket}$.

Let $\mathcal{P}_{0} \subset \mathcal{P}\left(\mathbb{W}_{0}\right), \ldots, \mathcal{P}_{T} \subset \mathcal{P}\left(\mathbb{W}_{T}\right)$, and $\left(\Upsilon_{t}\right)_{t \in \llbracket 0, T \rrbracket}$ be sequence of functions, each mapping $\mathcal{P}\left(\mathbb{W}_{t}\right)$ into $\overline{\mathbb{R}}$. Let $\left(\alpha_{t}\right)_{t \in \llbracket 0, T \rrbracket}$ be sequence of functions, each mapping $\mathbb{R}$ into $\mathbb{R}$. We set, for all $t \in \llbracket 0, T \rrbracket$,

$$
\varrho_{t, T}^{\text {cx }}\left(\left\{A_{s}\right\}_{t}^{T}\right)=\sup _{\mathbb{P}_{t} \in \mathcal{P}_{t}} \mathbb{E}_{\mathbb{P}_{t}}\left[\cdots \sup _{\mathbb{P}_{T} \in \mathcal{P}_{T}} \mathbb{E}_{\mathbb{P}_{T}}\left[\sum_{s=t}^{T}\left(\alpha_{s}\left(A_{s}\right)-\Upsilon_{s}\left(\mathbb{P}_{s}\right)\right)\right] \ldots\right],
$$

for any adapted uncertain process $\left\{A_{t}\right\}_{0}^{T}$, with the convention that $\alpha_{T}\left(c_{T}\right)=$ $c_{T}$.

Proposition 28. Time-consistency holds true for

- the dynamic uncertainty criterion $\left\{\varrho_{t, T}^{c x}\right\}_{t=0}^{T}$ given by (37),

- the Markov optimization problem

$$
\min _{\pi \in \Pi^{\text {ad }}} \varrho_{t, T}^{c x}\left(\left\{J_{t, s}^{x, \pi}\right\}_{s=t}^{T}\right), \quad \forall t \in \llbracket 0, T \rrbracket, \forall x \in \mathbb{X}_{t},
$$

where $J_{t, s}^{x, \pi}(w)$ is defined by 22 , as soon as there exists an admissible policy $\pi^{\sharp} \in \Pi^{\text {ad }}$ such that, for all $t \in \llbracket 0, T-1 \rrbracket$, for all $x \in \mathbb{X}_{t}$,

$$
\pi_{t}^{\sharp}(x) \in \underset{u \in U_{t}(x)}{\arg \min } \sup _{\mathbb{P}_{t} \in \mathcal{P}_{t}}\left\{\mathbb{E}_{\mathbb{P}_{t}}\left[\alpha_{t}\left(J_{t}(x, u, \cdot)\right)+V_{t+1} \circ f_{t}(x, u, \cdot)\right]-\Upsilon_{t}\left(\mathbb{P}_{t}\right)\right\},
$$

where the value functions are given by the following DPE

$$
\begin{aligned}
& V_{T}(x)=\sup _{\mathbb{P}_{T} \in \mathcal{P}_{T}} \mathbb{E}_{\mathbb{P}_{T}}\left[J_{T}(x, \cdot)\right]-\Upsilon_{T}\left(\mathbb{P}_{T}\right), \\
& V_{t}(x)=\min _{u \in U_{t}(x)} \sup _{\mathbb{P}_{t} \in \mathcal{P}_{t}}\left\{\mathbb { E } _ { \mathbb { P } _ { t } } \left[\alpha_{t}\left(J_{t}(x, u, \cdot)\right)\right.\right. \\
&\left.\left.\quad+V_{t+1} \circ f_{t}(x, u, \cdot)\right]-\Upsilon_{t}\left(\mathbb{P}_{t}\right)\right\} .
\end{aligned}
$$

Proof. We follow the proof of Proposition 27, where the one-step time-aggregators are defined by

$$
\Phi_{t}\left\{c_{t}, c_{t+1}\right\}=\alpha_{t}\left(c_{t}\right)+c_{t+1}, \forall t \in \llbracket 0, T-1 \rrbracket, \forall\left(c_{t}, c_{t+1}\right) \in \overline{\mathbb{R}}^{2},
$$

and the one-step uncertainty-aggregators are defined by

$$
\mathbb{G}_{t}\left[C_{t}\right]=\sup _{\mathbb{P}_{t} \in \mathcal{P}_{t}} \mathbb{E}_{\mathbb{P}_{t}}\left[C_{t}\right]-\Upsilon_{t}\left(\mathbb{P}_{t}\right), \quad \forall t \in \llbracket 0, T-1 \rrbracket, \quad \forall C_{t} \in \mathcal{F}\left(\mathbb{W}_{t} ; \overline{\mathbb{R}}\right) .
$$


We show that, for any $t \in \llbracket 0, T-1 \rrbracket$ and $s \in \llbracket t+1, T \rrbracket, \mathbb{G}_{s}$ commutes with $\Phi_{t}$ (we could also apply Proposition 31). Letting $c_{t}$ be an extended real number in $\overline{\mathbb{R}}$ and $C_{s}$ a function in $\mathcal{F}\left(\mathbb{W}_{s} ; \overline{\mathbb{R}}\right)$, we have

$$
\begin{aligned}
\mathbb{G}_{s}\left[\Phi_{t}\left\{c_{t}, C_{s}\right\}\right] & =\sup _{\mathbb{P}_{s} \in \mathcal{P}_{s}}\left\{\mathbb{E}_{\mathbb{P}_{s}}\left[\alpha\left(c_{t}\right)+C_{s}\right]-\Upsilon_{s}\left(\mathbb{P}_{s}\right)\right\} \text { by } 40 \mathrm{a} \text { and 40b } \\
& =\alpha_{t}\left(c_{t}\right)+\sup _{\mathbb{P}_{s} \in \mathcal{P}_{s}}\left\{\mathbb{E}_{\mathbb{P}_{s}}\left[C_{s}\right]-\Upsilon_{s}\left(\mathbb{P}_{s}\right)\right\} \\
& =\alpha_{t}\left(c_{t}\right)+\mathbb{G}_{s}\left[C_{s}\right] \text { by } 40 \mathrm{~b} \\
& =\Phi_{t}\left\{c_{t}, \mathbb{G}_{s}\left[C_{s}\right]\right\} \text { by } 40 \mathrm{a} .
\end{aligned}
$$

This ends the proof.

The one-step uncertainty-aggregators $\mathbb{G}_{t}$ in $40 \mathrm{~b}$ correspond to a convex risk measure (see [20]). Moreover, Proposition 31 shows that we could also consider any positive linear combination of suprema and infima of expectation.

\subsection{Worst-Case Risk Measures (Fear Operator)}

A special case of coherent risk measures consists of the worst case scenario operators, also called "fear operators". For this subclass of coherent risk measures, we show that time-consistency holds for a larger class of time-aggregators than the ones above.

For any $t \in \llbracket 0, T-1 \rrbracket$, let $\widetilde{\mathbb{W}}_{t}$ be a non empty subset of $\mathbb{W}_{t}$, and let $\Phi_{t}: \overline{\mathbb{R}}^{2} \rightarrow$ $\overline{\mathbb{R}}$ be a function which is continuous and non-decreasing in its second variable. We set, for all $t \in \llbracket 0, T \rrbracket$,

$$
\begin{array}{r}
\varrho_{t, T}^{\mathrm{wc}}\left(\left\{A_{s}\right\}_{t}^{T}\right)=\sup _{\left\{w_{s}\right\}_{t}^{T} \in \widetilde{\mathbb{W}}_{t} \times \cdots \times \widetilde{\mathbb{W}}_{T}} \Phi_{t}\left\{A_{t}\left(\left\{w_{s}\right\}_{t}^{T}\right), \Phi_{t+1}\{\cdots,\right. \\
\left.\left.\Phi_{T-1}\left\{A_{T-1}\left(w_{T-1}, w_{T}\right), A_{T}\left(w_{T}\right)\right\}\right\}\right\},
\end{array}
$$

for any adapted uncertain process $\left\{A_{t}\right\}_{0}^{T}$.

Note that $\varrho_{t, T}^{\mathrm{wc}}$ is the fear operator on the Cartesian product $\widetilde{\mathbb{W}}_{t} \times \cdots \times \widetilde{\mathbb{W}}_{T}$.

Proposition 29. Time-consistency holds true for

- the dynamic uncertainty criterion $\left\{\varrho_{t, T}^{w c}\right\}_{t=0}^{T}$ given by (41),

- the Markov optimization problem

$$
\min _{\pi \in \Pi^{\mathrm{ad}}} \varrho_{t, T}^{w c}\left(\left\{J_{t, s}^{x, \pi}\right\}_{s=t}^{T}\right),
$$

where $J_{t, s}^{x, \pi}(w)$ is defined by 22 , as soon as there exists an admissible policy $\pi^{\sharp} \in \Pi^{\text {ad }}$ such that, for all $t \in \llbracket 0, T-1 \rrbracket$, for all $x \in \mathbb{X}_{t}$,

$$
\pi_{t}^{\sharp}(x) \in \underset{u \in U_{t}(x)}{\arg \min } \sup _{w_{t} \in \widetilde{\mathbb{W}}_{t}} \Phi_{t}\left\{J_{t}\left(x, u, w_{t}\right), V_{t+1} \circ f_{t}\left(x, u, w_{t}\right)\right\},
$$


where the value functions are given by the following DPE

$$
\begin{aligned}
V_{T}(x) & =\sup _{w_{T} \in \widetilde{\mathbb{W}}_{T}} J_{T}\left(x, w_{T}\right), \\
V_{t}(x) & =\min _{u \in U_{t}(x)} \sup _{w_{t} \in \widetilde{\mathbb{W}}_{t}} \Phi_{t}\left\{J_{t}\left(x, u, w_{t}\right), V_{t+1} \circ f_{t}\left(x, u, w_{t}\right)\right\} .
\end{aligned}
$$

Proof. We follow the proof of Proposition 27, where

$$
\mathbb{G}_{t}\left[C_{t}\right]=\sup _{w_{t} \in \widetilde{\mathbb{W}}_{t}} C_{t}\left(w_{t}\right), \quad \forall t \in \llbracket 0, T-1 \rrbracket, \quad \forall C_{t} \in \mathcal{F}\left(\mathbb{W}_{t} ; \overline{\mathbb{R}}\right)
$$

We prove that, for any $t \in \llbracket 0, T-1 \rrbracket$ and $s \in \llbracket t+1, T \rrbracket, \mathbb{G}_{s}$ commutes with $\Phi_{t}$. Letting $c_{t}$ be an extended real number in $\overline{\mathbb{R}}$ and $C_{s}$ a function in $\mathcal{F}\left(\mathbb{W}_{s} ; \overline{\mathbb{R}}\right)$, we have

$$
\begin{array}{rlrl}
\mathbb{G}_{s}\left[\Phi_{t}\left\{c_{t}, C_{s}\right\}\right] & =\sup _{w_{s} \in \mathbb{\mathbb { W }}_{s}}\left[\Phi_{t}\left\{c_{t}, C_{s}\left(w_{s}\right)\right\}\right] \\
& =\Phi_{t}\left\{c_{t}, \sup _{w \in \widetilde{\mathbb{W}}_{s}}\left[C_{s}\left(w_{s}\right)\right]\right\} & \text { by } \\
& =\Phi_{t}\left\{c_{t}, \mathbb{G}_{s}\left[C_{s}\right]\right\} & \text { by continuity of } \Phi_{t}\left\{c_{t}, \cdot\right\},
\end{array}
$$

${ }_{435}$ This ends the proof.

\subsection{Examples}

State-dependent discounting and beliefs

We consider the following long term investment problem. Let $J_{t}\left(x_{t}, u_{t}, w_{t}\right)$ be the cost incurred at time $t$ in the state $x_{t}$, under decision $u_{t}$ and uncertainty $w_{t}$. The state $x_{t}$ includes economic indicators, one of them affecting the discount factor $e^{-r_{t}\left(x_{t}\right)}$. Hence, the time-aggregation of the cost process is given by

$$
\sum_{t=0}^{T-1} e^{-r_{t}\left(\boldsymbol{I}_{t}\right)} J_{t}\left(\boldsymbol{X}_{t}, \boldsymbol{U}_{t}, \boldsymbol{W}_{t}\right) .
$$

We suppose that the one-step uncertainty aggregators are coherent risk measures

$$
\mathbb{G}_{t}^{x_{t}}[\cdot]=\sup _{\mathbb{Q} \in \mathcal{P}\left(x_{t}\right)} \mathbb{E}_{\mathbb{Q}}[\cdot]
$$

where the probability set $\mathcal{P}\left(x_{t}\right)$ of beliefs is allowed to depend on the economic indicators in $x_{t}$.

Such an optimization problem, where both discounting and beliefs depend on the state, falls into the framework developped in 4.1 in its Markovian version. 
Non-additive time preferences

In environmental economics literature, when time spans across generations (like with climate change issues), scholars discuss the use of additive time preferences. Indeed, additivity implies possible compensations between distant generations, and discounting can lead to myopic decisions [21, 22]. The so-called Rawls or maximin criterion 23 ]

$$
\min \left\{J_{0}\left(x_{0}, u_{0}, w_{0}\right), \cdots, J_{T-1}\left(x_{T-1}, u_{T-1}, w_{T-1}\right)\right\}
$$

is a possible alternative, which can be obtained by aggregation of one-step timeaggregators $\Phi_{t}\left\{c_{t}, c_{t+1}\right\}=\max \left\{c_{t}, c_{t+1}\right\}$. Now, when the uncertainty aggregator is the worst-case operator over the Cartesian product $\widetilde{\mathbb{W}}_{t} \times \cdots \times \widetilde{\mathbb{W}}_{T-1}$, the resulting optimization problem falls into the framework developped in 4.3 .

\section{Risk-sensitive optimization}

We consider a family of risk-sensitive optimization problems

$$
\begin{aligned}
\min & \log \left(\mathbb{E}\left[\exp \left(\sum_{t=t_{0}}^{T-1} J_{t}\left(\boldsymbol{X}_{t}, \boldsymbol{U}_{t}, \boldsymbol{W}_{t}\right)\right)\right]\right) \\
& \boldsymbol{X}_{t+1}=f_{t}\left(\boldsymbol{X}_{t}, \boldsymbol{U}_{t}, \boldsymbol{W}_{t}\right), \quad \boldsymbol{X}_{t_{0}}=x_{0} \\
& \boldsymbol{U}_{t} \in U_{t}\left(\boldsymbol{X}_{t}\right) .
\end{aligned}
$$

This family is time-consistent, after an equivalent reformulation as follows. First, as the function $\log$ is increasing, it is equivalent to minimize its argument. Second, denoting $\tilde{J}_{t}\left(\boldsymbol{X}_{t}, \boldsymbol{U}_{t}, \boldsymbol{W}_{t}\right)=\exp \left(J_{t}\left(\boldsymbol{X}_{t}, \boldsymbol{U}_{t}, \boldsymbol{W}_{t}\right)\right)$, we arrive at the following optimization problem

$$
\begin{aligned}
\min & \mathbb{E}\left[\prod_{t=t_{0}}^{T-1}\left(\tilde{J}_{t}\left(\boldsymbol{X}_{t}, \boldsymbol{U}_{t}, \boldsymbol{W}_{t}\right)\right)\right] \\
& \boldsymbol{X}_{t+1}=f_{t}\left(\boldsymbol{X}_{t}, \boldsymbol{U}_{t}, \boldsymbol{W}_{t}\right), \quad \boldsymbol{X}_{t_{0}}=x_{0} \\
& \boldsymbol{U}_{t} \in U_{t}\left(\boldsymbol{X}_{t}\right)
\end{aligned}
$$

Hence, by changing costs, we are falling into the setting of Proposition 27, and we can write a time-consistent Markov decision problem equivalent to Problem 48.

\section{Conclusion}

We have provided basic ingredients - one-step time and uncertainty aggregators - to make up dynamic uncertainty criteria and corresponding intertemporal optimization problems under uncertainty. Nested criteria carry the time-consistency property in the very manner they are built, only relying on monotonicity property. Block-aggregated are more natural candidates than 
nested ones as dynamic uncertainty criteria. However, we prove that they display time-consistency at the additional price of commutation between one-step time and uncertainty aggregators.

Thus equipped, we have tools to cook up time-consistent dynamic uncertainty criteria and intertemporal optimization problems under uncertainty. Moreover, our framework extends to the Markovian setting, where the one-step time and uncertainty aggregators are parametrized by the state, that follows a control dynamical system. This is how our results cover a large class of applications. However, our results also point to the fact that, in practice, time-consistency 465 is mostly related to linearity and expectation (see [18 for some comments on the subject). Other ways to obtain time-consistent formulation consist in using either a worst case approach, or a nested formulation from the beginning.

We think that an interesting question for further research is the following. Given a dynamic risk measure or an intertemporal optimization problem under uncertainty, can we identify a state or a new state such that time-consistency holds true with this new information setting? A first set of answers can be found in 24]. We think that our analysis provides insight to look after the properties expected from such a state.

\section{Appendix A. Constructing new commuting aggregators}

We show that we can construct new commuting one-step uncertainty aggregators either as linear combination of suprema or infima of one-step commuting aggregators.

Proposition 30. Let $\Phi$ be a one-step time-aggregator and $\left(\mathbb{G}_{t}^{i}\right)_{i \in I}$ be a family of one-step uncertainty aggregators. Suppose that $\mathbb{G}_{t}^{i}$ commutes with $\Phi$, for all ${ }_{480} i \in I$, and that

- either, for all $c \in \overline{\mathbb{R}}, \Phi\{c, \cdot\}$ is continuous and non-decreasing;

- or, for all $C_{t} \in \mathcal{F}\left(\mathbb{W}_{t}, \overline{\mathbb{R}}\right), \sup _{i \in I} \mathbb{G}_{t}^{i}\left[C_{t}\right]$ is attained (always true for $I$ finite).

Then, the one-step uncertainty-aggregator $\sup _{i \in I} \mathbb{G}_{t}^{i}$ commutes with $\Phi$, and so

does $\inf _{i \in I} \mathbb{G}_{t}^{i}$, provided $\inf _{i \in I} \mathbb{G}_{t}^{i}$ never takes the value $-\infty$.

Proof. We consider the supremum case, the infimum being similar. For any $\left(c, C_{t}\right) \in \overline{\mathbb{R}} \times \mathcal{F}\left(\mathbb{W}_{t}, \overline{\mathbb{R}}\right)$, we have

$$
\begin{aligned}
\overline{\mathbb{G}}_{t}\left[\Phi\left\{c, C_{t}\right\}\right] & =\sup _{i \in I} \mathbb{G}_{t}^{i}\left[\Phi\left\{c, C_{t}\right\}\right] \text { by definition of } \overline{\mathbb{G}}_{t} \\
& =\sup _{i \in I} \Phi\left\{c, \mathbb{G}_{t}^{i}\left[C_{t}\right]\right\} \text { by commutation of } \mathbb{G}_{i} \text { and } \Phi \\
& =\Phi\left\{c, \sup _{i \in I} \mathbb{G}_{t}^{i}\left[C_{t}\right]\right\} .
\end{aligned}
$$


The last equality being obtained either because the supremum is attained or by continuity.

The following Proposition allows to build uncertainty aggregators commuting with affine or linear (in the second variable) time-aggregator.

Proposition 31. Let $\Phi$ be a one-step time-aggregator and $\left(\mathbb{G}_{t}^{i}\right)_{i \in I}$ be a family of one-step uncertainty aggregators. Suppose that for all $i \in I$, the timestep uncertainty aggregator $\mathbb{G}_{i}$ commutes with the time-step time aggregator $\Phi$. For $j \in \llbracket 1, n \rrbracket$, let $\underline{I}_{j} \subset I$, and $\bar{I}_{j} \subset I$, be families of subsets of $I$, and $\left(\left\{\underline{\theta}_{j}\right\}_{j \in \llbracket 1, N \rrbracket},\left\{\bar{\theta}_{j}\right\}_{j \in \llbracket 1, N \rrbracket}\right)$ be non-negative scalars. We define

$$
\overline{\mathbb{G}}=\sum_{j=1}^{n} \underline{\theta}_{j} \inf _{i \in \underline{I}_{j}} \mathbb{G}_{t}^{i}+\sum_{j=1}^{n} \bar{\theta}_{j} \sup _{i \in \bar{I}_{j}} \mathbb{G}_{t}^{i}
$$

- If $\Phi$ is affine in its second variable, that is, if

$$
\Phi\{c, d\}=\alpha(c)+\beta(c) d,
$$
tion $\overline{\mathbb{G}}$ of infima and suprema of subfamilies of $\left\{\mathbb{G}_{t}^{i}\right\}_{i \in I}$ commutes with $\Phi$, provided $\inf _{i \in \underline{I}_{j}} \mathbb{G}_{t}^{i}$ never takes the value $-\infty$.

- If $\Phi$ is linear in its second variable, that is, if

$$
\Phi\{c, d\}=\beta(c) d,
$$

then the linear combination $\overline{\mathbb{G}}$ of infima and suprema of subfamilies of $\left\{\mathbb{G}_{t}^{i}\right\}_{i \in I}$ commutes with $\Phi$.

Proof. Assume that $\Phi$ is given by $(A .2)$, and define

$$
\overline{\mathbb{G}}=\sum_{j=1}^{n} \underline{\theta}_{j} \inf _{i \in \underline{I}_{j}} \mathbb{G}_{t}^{i}+\sum_{j=1}^{n} \bar{\theta}_{j} \sup _{i \in \bar{I}_{j}} \mathbb{G}_{t}^{i}
$$

Then, we have

$$
\begin{aligned}
\overline{\mathbb{G}}\left[\Phi\left\{c, \boldsymbol{C}_{t}\right\}\right] & =\left(\sum_{j=1}^{n} \underline{\theta}_{j} \inf _{i \in \underline{I}_{j}} \mathbb{G}_{t}^{i}+\sum_{j=1}^{n} \bar{\theta}_{j} \sup _{i \in \bar{I}_{j}} \mathbb{G}_{t}^{i}\right)\left[\Phi\left\{c, \boldsymbol{C}_{t}\right\}\right] \\
& =\sum_{j=1}^{n} \underline{\theta}_{j} \inf _{i \in \underline{I}_{j}} \mathbb{G}_{t}^{i}\left[\alpha(c)+\beta(c) \boldsymbol{C}_{t}\right]+\sum_{j=1}^{n} \bar{\theta}_{j} \sup _{i \in \bar{I}_{j}} \mathbb{G}_{t}^{i}\left[\alpha(c)+\beta(c) \boldsymbol{C}_{t}\right] \\
& =\sum_{j=1}^{n} \underline{\theta}_{j}\left(\alpha(c)+\beta(c) \inf _{i \in \underline{I}_{j}} \mathbb{G}_{t}^{i}\left[\boldsymbol{C}_{t}\right]\right)+\sum_{j=1}^{n} \bar{\theta}_{j}\left(\alpha(c)+\beta(c) \sup _{i \in \bar{I}_{j}} \mathbb{G}_{t}^{i}\left[\boldsymbol{C}_{t}\right]\right) \\
& =\alpha(c)+\beta(c) \sum_{j=1}^{n} \underline{\theta}_{j} \inf _{i \in \underline{I}_{j}} \mathbb{G}_{t}^{i}\left[\boldsymbol{C}_{t}\right]+\sum_{j=1}^{n} \bar{\theta}_{j} \sup _{i \in \bar{I}_{j}} \mathbb{G}_{t}^{i}\left[\boldsymbol{C}_{t}\right]=\Phi\left\{c, \overline{\mathbb{G}}\left[\boldsymbol{C}_{t}\right]\right\},
\end{aligned}
$$

where the last equality is obtained either because the coefficient $\theta_{j}$ sum to one (affine case) or because $\alpha$ is equal to zero (linear case). 


\section{Appendix B. Technical Proofs}

Appendix B.1. Proof of Lemma 12

Proof. We prove by induction that

$$
\left(\stackrel{\bullet}{s=t}_{\vec{G}}^{T} \mathbb{G}_{s}\right)\left[\Phi_{r}\left\{c, D_{t}\right\}\right]=\Phi_{r}\left\{c,\left(\stackrel{T}{\bullet_{s=t}} \mathbb{G}_{s}\right)\left[D_{t}\right]\right\}, \quad \forall 0 \leq r<t \leq T,
$$

for any extended scalar $c \in \overline{\mathbb{R}}$ and any function $D_{t} \in \mathcal{F}\left(\mathbb{W}_{[t: T]} ; \overline{\mathbb{R}}\right)$. For $t \in$ $\llbracket 1, T \rrbracket$, let $\left(H_{t}\right)$ be the following assertion

$$
\begin{aligned}
& \left(H_{t}\right): \forall r \in \llbracket 0, t-1 \rrbracket, \forall c \in \overline{\mathbb{R}}, \forall D_{t} \in \mathcal{F}\left(\mathbb{W}_{[t: T]} ; \overline{\mathbb{R}}\right), \\
& \left(\stackrel{T}{\cdot} \mathbb{G}_{s=t}\right)\left[\Phi_{r}\left\{c, D_{t}\right\}\right]=\Phi_{r}\left\{c,\left(\stackrel{T}{\cdot} \mathbb{G}_{s=t}\right)\left[D_{t}\right]\right\} .
\end{aligned}
$$

The assertion $\left(H_{T}\right)$ coincides with the property that, for any $0 \leq r<T, \mathbb{G}_{T}$ commutes with $\Phi_{r}$ (apply (14) where $t=T, \Phi=\Phi_{r}$ ), and hence holds true.

Now, suppose that $\left(H_{t+1}\right)$ holds true. Let $r<t, c \in \overline{\mathbb{R}}$ and $D_{t} \in \mathcal{F}\left(\mathbb{W}_{[t: T]} ; \overline{\mathbb{R}}\right)$. We have

$$
\begin{aligned}
& \left(\stackrel{\bullet}{\cdot} \mathbb{G}_{s=t}\right)\left[\Phi_{r}\left\{c, D_{t}\right\}\right] \\
& =\mathbb{G}_{t}\left[w_{t} \mapsto\left(\stackrel{T}{\stackrel{\bullet}{\bullet} \mathbb{G}_{s}}\right)\left[w_{[t+1: T]} \mapsto \Phi_{r}\left\{c, D_{t}\left(w_{t}, w_{[t+1: T]}\right)\right\}\right]\right], \\
& =\mathbb{G}_{t}\left[w_{t} \mapsto \Phi_{r}\left\{c,\left(\underset{s=t+1}{\stackrel{T}{\ominus}} \mathbb{G}_{s}\right)\left[w_{[t+1: T]} \mapsto D_{t}\left(w_{t}, w_{[t+1: T]}\right)\right]\right\}\right] \\
& \text { by }\left(H_{t+1}\right) \text { since } r<t<t+1 \text {, } \\
& \text { and where, for all } w_{t}, D_{t+1}: w_{[t+1: T]} \mapsto D_{t}\left(w_{t}, w_{[t+1: T]}\right) \in \mathcal{F}\left(\mathbb{W}_{[t: T]} ; \overline{\mathbb{R}}\right) \text {, } \\
& =\Phi_{r}\left\{c, \mathbb{G}_{t}\left[w_{t} \mapsto\left(\underset{s=t+1}{\stackrel{T}{\bullet}} \mathbb{G}_{s}\right)\left[w_{[t+1: T]} \mapsto D_{t}\left(w_{t}, w_{[t+1: T]}\right)\right]\right]\right\}, \\
& =\Phi_{r}\left\{c,\left(\stackrel{\unrhd_{s=t}^{T} \mathbb{G}_{s}}{)}\left[D_{t}\right]\right\}\right.
\end{aligned}
$$

This ends the induction, hence the proof of B.1 which leads to 16 .

\section{Appendix B.2. Proof of Proposition 22}

Proof. In the proof, we denote by $V_{t}$ the sequence of function given by $(30)$. Let $\pi \in \Pi^{\text {ad }}$ be a policy. For any $t \in \llbracket 0, T \rrbracket$, we define $V_{t}^{\pi}(x)$ as the intertemporal 
cost from time $t$ to time $T$ when following policy $\pi$ starting from state $x$ :

$$
V_{t}^{\pi}(x)=\varrho_{t, T}^{N}\left(\left\{J_{t, s}^{x, \pi}\right\}_{s=t}^{T}\right), \quad \forall t \in \llbracket 0, T \rrbracket, \forall x \in \mathbb{X}_{t} .
$$

This expression is well defined because $J_{t, s}^{x, \pi}: \mathbb{W}_{[t: s]} \rightarrow \overline{\mathbb{R}}$, for $s \in \llbracket t, T \rrbracket$ by (23).

First, we show that the functions $\left\{V_{t}^{\pi}\right\}_{t=0}^{T}$ satisfy a backward equation "à la Bellman":

$V_{t}^{\pi}(x)=\mathbb{G}_{t}\left[\Phi_{t}\left\{J_{t}\left(x, \pi_{t}(x), \cdot\right), V_{t+1}^{\pi} \circ f_{t}\left(x, \pi_{t}(x), \cdot\right)\right\}\right], \forall t \in \llbracket 0, T-1 \rrbracket, \forall x \in \mathbb{X}_{t}$.

Indeed, we have,

$$
\begin{aligned}
V_{T}^{\pi}(x) & =\varrho_{T, T}^{N}\left(J_{T, T}^{x, \pi}\right) & & \text { by the definition } \mathrm{B} .3 \text { of } V_{T}^{\pi}(x), \\
& =\varrho_{T, T}^{N}\left(J_{T}(x, \cdot)\right) & & \text { by } 22 \text { that defines } J_{T, T}^{x, \pi}, \\
& =\mathbb{G}_{T}\left[J_{T}(x, \cdot)\right] & & \text { by the definition } 11 \mathrm{a}) \text { of } \varrho_{T}^{N}, \\
& =\mathbb{G}_{T}\left[J_{T}(x, \cdot)\right] & & \text { by Definition of } \mathbb{G}_{T} .
\end{aligned}
$$

We also have, for $t \in \llbracket 0, T-1 \rrbracket$,

$$
\begin{array}{rlrl}
V_{t}^{\pi}(x) & =\varrho_{t, T}^{N}\left(\left\{J_{t, s}^{x, \pi}\right\}_{s=t}^{T}\right) & & \text { by (B.3) } \\
& =\mathbb{G}_{t}\left[\Phi_{t}\left\{J_{t, t}^{x, \pi}, \varrho_{t+1, T}^{N}\left(\left\{J_{t, s}^{x, \pi}\right\}_{s=t+1}^{T}\right)\right\}\right] & & \text { by 11b } \\
& =\mathbb{G}_{t}\left[\Phi_{t}\left\{J_{t, t}^{x, \pi}, \varrho_{t+1, T}^{N}\left(\left\{J_{t+1, s}^{f_{t}\left(x, \pi_{t}(x), \cdot\right), \pi}\right\}_{s=t+1}^{T}\right)\right\}\right] & & \text { by (24) } \\
& =\mathbb{G}_{t}\left[\Phi_{t}\left\{J_{t, t}^{x, \pi}, V_{t+1}^{\pi} \circ f_{t}\left(x, \pi_{t}(x), \cdot\right)\right\}\right] & & \text { by (B.3) } \\
& =\mathbb{G}_{t}\left[\Phi_{t}\left\{J_{t}\left(x, \pi_{t}(x), \cdot\right), V_{t+1}^{\pi} \circ f_{t}\left(x, \pi_{t}(x), \cdot\right)\right\}\right] & & \text { by (24) } \\
& =\mathbb{G}_{t}\left[\Phi_{t}\left\{J_{t}\left(x, \pi_{t}(x), \cdot\right), V_{t+1}^{\pi} \circ f_{t}\left(x, \pi_{t}(x), \cdot\right)\right\}\right] . &
\end{array}
$$

Second, we show that $V_{t}(x)$, as defined in $(30)$ is lower than the value of the optimization problem $\mathfrak{P}_{t}^{N}(x)$ in (28). For this purpose, we denote by $\left(H_{t}\right)$ the following assertion

$$
\left(H_{t}\right): \quad \forall x \in \mathbb{X}_{t}, \forall \pi \in \Pi^{\mathrm{ad}}, \quad V_{t}(x) \leq V_{t}^{\pi}(x) .
$$

By definition of $V_{T}^{\pi}(x)$ in $(\mathrm{B} .3)$ and of $V_{T}(x)$ in $\left.30 \mathrm{a}\right)$, assertion $\left(H_{T}\right)$ is true.

Now, assume that $\left(H_{t+1}\right)$ holds true. Let $x$ be an element of $\mathbb{X}_{t}$. Then, by definition of $V_{t}(x)$ in $(30 \mathrm{~b})$, we obtain

$$
V_{t}(x) \leq \inf _{\pi \in \Pi^{\text {ad }}} \mathbb{G}_{t}\left[\Phi_{t}\left\{J_{t}\left(x, \pi_{t}(x), \cdot\right), V_{t+1} \circ f_{t}\left(x, \pi_{t}(x), \cdot\right)\right\}\right],
$$


since, for all $\pi \in \Pi^{\text {ad }}$ we have $\pi_{t}(x) \in U_{t}(x)$. By $\left(H_{t+1}\right)$ we have, for any $\pi \in \Pi^{\mathrm{ad}}$

$$
V_{t+1} \circ f_{t}\left(x, \pi_{t}(x), \cdot\right) \leq V_{t+1}^{\pi} \circ f_{t}\left(x, \pi_{t}(x), \cdot\right) .
$$

From monotonicity of $\Phi_{t}$ and monotonicity of $\mathbb{G}_{t}$, we deduce:

$$
\begin{aligned}
\mathbb{G}_{t} & {\left[\Phi_{t}\left\{J_{t}\left(x, \pi_{t}(x), \cdot\right), V_{t+1} \circ f_{t}\left(x, \pi_{t}(x), \cdot\right)\right\}\right] } \\
& \leq \mathbb{G}_{t}\left[\Phi_{t}\left\{J_{t}\left(x, \pi_{t}(x), \cdot\right), V_{t+1}^{\pi} \circ f_{t}\left(x, \pi_{t}(x), \cdot\right)\right\}\right] .
\end{aligned}
$$

We obtain:

$$
\begin{array}{rlr}
V_{t}(x) & \leq \inf _{\pi \in \Pi^{\mathrm{ad}}} \mathbb{G}_{t}\left[\Phi_{t}\left\{J_{t}\left(x, \pi_{t}(x), \cdot\right), V_{t+1} \circ f_{t}\left(x, \pi_{t}(x), \cdot\right)\right\}\right] \quad \text { by B.5, } \\
& \leq \inf _{\pi \in \Pi^{\mathrm{ad}}} \mathbb{G}_{t}\left[\Phi_{t}\left\{J_{t}\left(x, \pi_{t}(x), \cdot\right), V_{t}^{\pi} \circ f_{t+1}\left(x, \pi_{t}(x), \cdot\right)\right\}\right] \quad \text { by B.6. } \\
& =\inf _{\pi \in \Pi^{\mathrm{ad}}} V_{t}^{\pi}(x) \text { by the definition B.3 of } V_{t}^{\pi}(x) .
\end{array}
$$

Hence, assertion $\left(H_{t}\right)$ holds true.

Third, we show that the lower bound $V_{t}(x)$ for the value of the optimization problem $\mathfrak{P}_{t}^{N}(x)$ is achieved for the policy $\pi^{\sharp}$ in 290 . For this purpose, we consider the following assertion

$$
\left(H_{t}^{\prime}\right): \quad \forall x \in \mathbb{X}_{t}, \quad V_{t}^{\pi^{\sharp}}(x)=V_{t}(x) .
$$

By definition of $V_{T}^{\pi^{\sharp}}(x)$ in (B.3) and of $V_{T}(x)$ in 30a), $\left(H_{T}^{\prime}\right)$ holds true. For $t \in \llbracket 0, T-1 \rrbracket$, assume that $\left(H_{t+1}^{\prime}\right)$ holds true. Let $x$ be in $\mathbb{X}_{t}$. We have

$$
\begin{aligned}
V_{t}(x) & =\mathbb{G}_{t}\left[\Phi_{t}\left\{J_{t}\left(x, \pi_{t}^{\sharp}(x), \cdot\right), V_{t+1} \circ f_{t}\left(x, \pi_{t}^{\sharp}(x), \cdot\right)\right\}\right] & & \text { by definition of } \pi^{\sharp} \text { in } 29 ., \\
& =\mathbb{G}_{t}\left[\Phi_{t}\left\{J_{t}\left(x, \pi_{t}^{\sharp}(x), \cdot\right), V_{t+1}^{\pi^{\sharp}} \circ f_{t}\left(x, \pi_{t}^{\sharp}(x), \cdot\right)\right\}\right] & & \text { by }\left(H_{t+1}^{\prime}\right) \\
& =V_{t}^{\pi^{\sharp}}(x) & & \text { by B.3. }
\end{aligned}
$$

Hence $\left(H_{t}^{\prime}\right)$ holds true, and the proof is complete by induction.

\section{References}

[1] B. Rudloff, A. Street, D. M. Valladão, Time consistency and risk averse dynamic decision models: Definition, interpretation and practical consequences, European Journal of Operational Research 234 (3) (2014) 743750.

[2] B. Peleg, M. Yaari, On the existence of a consistent course of action when tastes are changing, Review of Economic Studies 40 (3) (1973) 391-401. 
[3] R. H. Strotz, Myopia and inconsistency in dynamic utility maximization, The Review of Economic Studies 23 (3) (1955-1956) 165-180.

[4] S. Dreyfus, Richard Bellman on the birth of dynamic programming, Operations Research 50 (1) (2002) 48-51.

[5] P. Hammond, Changing tastes and coherent dynamic choice, The Review of Economic Studies 43 (1) (1976) 159-173.

[6] D. Kreps, E. Porteus, Temporal resolution of uncertainty and dynamic choice theory, Econometrica: journal of the Econometric Society 46 (1) (1978) 185-200.

[7] P. Carpentier, J.-P. Chancelier, G. Cohen, M. De Lara, P. Girardeau, Dynamic consistency for stochastic optimal control problems, Annals of Operations Research 200 (1) (2012) 247-263.

[8] F. Riedel, Dynamic coherent risk measures, Stochastic Processes and their Applications 112 (2) (2004) 185-200.

[9] K. Detlefsen, G. Scandolo, Conditional and dynamic convex risk measures, Finance and Stochastics 9 (4) (2005) 539-561.

[10] P. Cheridito, F. Delbaen, M. Kupper, Dynamic monetary risk measures for bounded discrete-time processes, Electronic Journal of Probability 11 (3) (2006) 57-106.

[11] P. Artzner, F. Delbaen, J.-M. Eber, D. Heath, H. Ku, Coherent multiperiod risk adjusted values and Bellmans principle, Annals of Operations Research 152 (1) (2007) 5-22.

[12] P. Cheridito, M. Kupper, Composition of time-consistent dynamic monetary risk measures in discrete time, International Journal of Theoretical and Applied Finance 14 (01) (2011) 137-162.

[13] R. E. Bellman, Dynamic Programming, Princeton University Press, Princeton, N.J., 1957.

[14] P. Whittle, Optimization over time, John Wiley \& Sons, Inc. New York, NY, USA, 1982.

[15] D. Bertsekas, Dynamic Programming and Optimal Control, 2nd Edition, Athena Scientific, Belmont, Massachusets, 2000, volumes 1 and 2.

[16] V. Leclère, Contribution to decomposition methods in stochastic optimisation, PhD dissertation, École des Ponts ParisTech, Marne-la-Vallée, France (2014).

[17] A. Ruszczyski, Risk-averse dynamic programming for Markov decision processes, Mathematical Programming 125 (2010) 235-261. 
[18] A. Shapiro, Minimax and risk averse multistage stochastic programming, European Journal of Operational Research 219 (3) (2012) 719-726.

[19] P. Artzner, F. Delbaen, J. Eber, D. Heath, Coherent measures of risk, Mathematical Finance 9 (1999) 203-228.

[20] H. Föllmer, A. Schied, Convex measures of risk and trading constraints, Finance and Stochastics 6 (2002) 429-447.

[21] C. W. Clark, Mathematical Bioeconomics, 2nd Edition, Wiley, New York, 1990.

[22] M. L. Weitzman, A review of the Stern review on the economics of climate change, Journal of Economic Literature 45 (3) (2007) 703-724.

[23] M. De Lara, L. Doyen, Sustainable management of natural resources, Springer, 2008.

[24] G. Pflug, A. Pichler, On dynamic decomposition of multistage stochastic programs, Optimization Online preprint 2 (2012) 11. 